


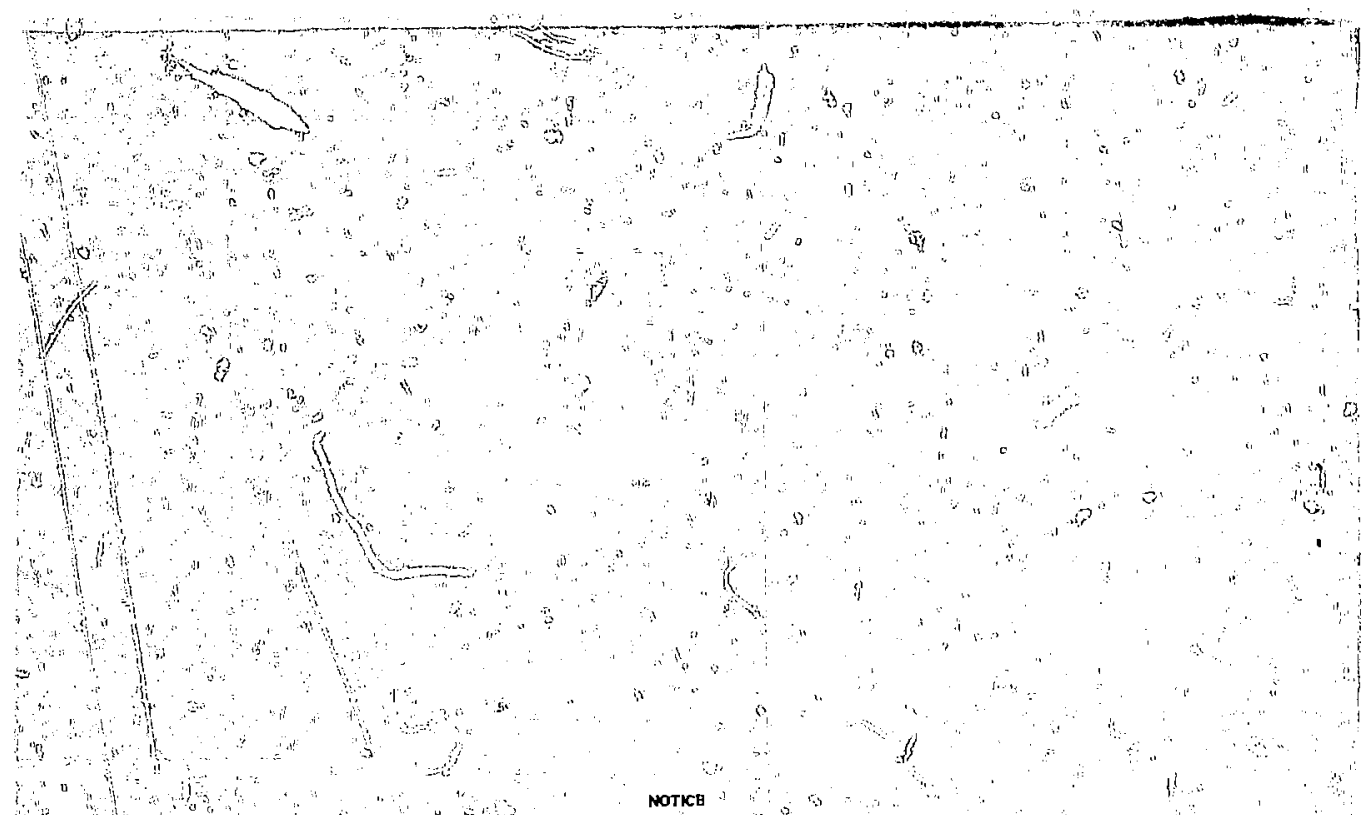

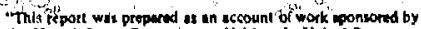
the United Stated Goveminent. Neliher the United States not the United state Governinan. Nellier the Units stated not

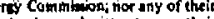
employess, not any of tikly contratlon, suboontractors, or their employoed, makes any wartapty, expres or inplied, or asumes

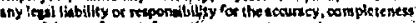
or teafulnes of any information, apinalus product or procest disdoud, or neptesente that is uac would ant infringe privitety. ownod riphers."

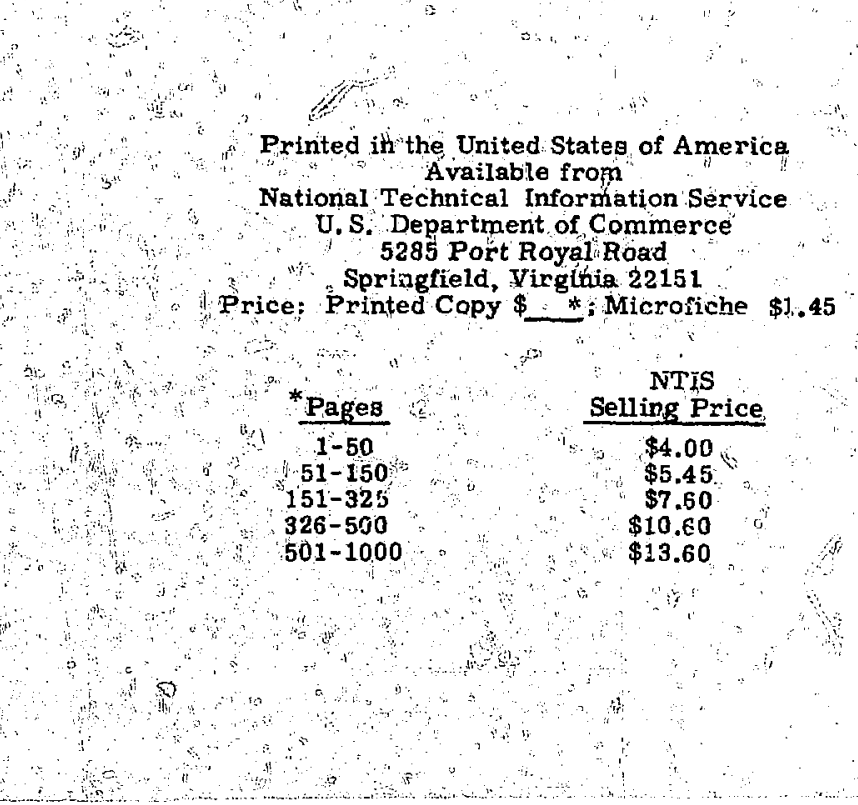


TID-4500, UC-34d

Physics - Particles and Fields

\section{近 \\ LAWRENCE IVEAMORE LABORATOAY

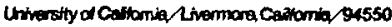

\section{UCRL-51631 Pt. I \\ INTERACTION OF INTENSE MICROWAVE RADIATION AND FULLY IONIZED HYDROGEN PLASMA Part 1: High-Density System}

Donald L. Ensley

MS. date: Jul: 26,1974

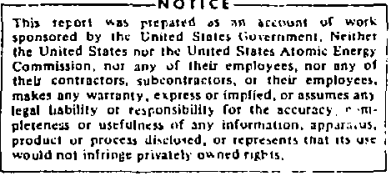

管 


\section{Contents}

Abstract . . . . . . . . . . . . . . . . . . . . . . . . . . . . . 1

Introduction. . . . . . . . . . . . . . . . . . . . . . . . . . . . . . . . 1

List of Relevant Eqtutions . . . . . . . . . . . . . . . . . . . . . . 1

Symbols . . . . . . . . . . . . . . . . . . . , . . . . . . . . . . I

Plasma-Blicowave lnteractions . . . . . . . . . . . . . . . . . . . . . . . . . . . . 3

Individual Particle Behavior . . . . . . . . . . . . . . . . . . . . . . . . . . . . . . 3

Stimulated Bremsstrallilung . . . . . . . . . . . . . . . . . . . . . . . . . . . 3

Stochastic or Transit-Time Eflects . . . . . . . . . . . . . . . . . . . . . . 3

Collective Effects . . . . . . . . . . . . . . . . . . . . . . . . . . . . . . . . . . . 3

"Shock" or Collective Collisionless Heating Due to Physically Displacing the

Plasma Coron at Twice Field Frequency . . . . . . . . . . . . . . . . . . . . . . . 3

Phasmit-Wave Excitation and Instabilities . . . . . . . . . . . . . . . . . . . . . . 4

Hydroradiation Surface Instabilities . . . . . . . . . . . . . . . . . . . . . . . . . 4

Higl1-Deusity Plasma . . . . . . . . . . . . . . . . . . . . . . . . . . . . . . . . . 4

Sell-Consistent Fieids .. High-Frequency Skin . . . . . . . . . . . . . . . . . . . . . . 4

Role of Fluctuations on the Skin Depth . . . . . . . . . . . . . . . . . . . . . . . . . . s

Role of Collisions on the Skin Field . . . . . . . . . . . . . . . . . . . . . . 6

Self-Consistent Solutions . . . . . . . . . . . . . . . . . . . , . 7

Applicution to High-Density Plasma Heating . . . . . . . . . . . . . . . . . . . . . . . . 7

Stimulated Inverse Bremsstrablung (Skin Effect) . . . . . . . . . . . . . . . . . 8

Wave-Poricle Heating . . . . . . . . . . . . . . . . . . . . , s

Stochastic Heating . . . . . . . . . . . . . . . . . . , . . . . . . . . . . 9

Application to Bootstrap Burn Conditions . . . . . . . . . . . . . . . . . 10

Appendix A: Stimulated Bremsstrahlung . . . . . . . . . . . . . . . . . . . 12

1. A Classical Derivation of Stimulated Bremsstrahlung . . . . . . . . . . . . . . . . . . 12

II. Relation to the Quantum Treatment of Stimulated Bremsstrahlung . . . . . . . . . . . 19

III. Collisional Microwave Skin Heating . . . . . . . . . . . . . . . . . . . . . . . . 22

Appendix B: Collective Collisionless lon Heating . . . . . . . . . . . . . . . . . . . . . . . . . . 74

Appendix C: Relativistic Quasi-Potertials . . . . . . . . . . . . . . . . . . . . . . . 28

A ppendix D: Plasma Hydroradiation Stabihty . . . . . . . . . . . . . . . . . . . . . . . 30

Fiek Perturbation . . . . . . . . . . . . . . . . . . . . . . . . . . . 30

Plasma Particle Pressure . . . . . . . . . . . . . . . . . . . . . . . . . . . . . . . 33

Dispersion Rblation fo: Surface Waves . . . . . . . . . . . . . . . . . . . . . . . . . . 34

Ablative Stability . . . . . . . . . . . . . . . . . . . . . 35 
Appendix E: Derivation of the Self-Consistent Field Equation for

a Microwave Corona . . . . . . . . . . . . . . . . . . . . . . . . . . . . . . . . . 37

Acknowledgments . . . . . . . . . . . . . . . . . . . . . . . . . . . . . . . 40

Bibliograplıy . . . . . . . . . . . . . . . . . . . . . . . . . . . . . . 40 


\title{
INTERACTION OF INTENSE MICROWAVE RADIATION AND FULLY IONIZED HYDROGEN PLASMA \\ Part 1: High-Density System
}

\begin{abstract}
The inteiaction of intense standing-wave radiation alm it fully ionized plasma is insestigided for $\omega / \omega_{p e}<I$. The high-density case is treated here, for which $\nu_{\mathrm{e}} / \omega \geq 1$ at corona densities satisfying the confilkment relition, but ior which there is no average confinement. It is demonstruted that under cervin realizable circunstances tire energy-absorption time of the plisma is less than the inertial disussimbly time, and that the radiation/plasma interface is stable for perturbations less than the exter!al field radiation wavelengtl.
\end{abstract}

\section{Introduction}

The application of microwave confinement and heating to controlled fusion has been historieblly the subject of much research in the Soviet Union and Europe, but until recently it lias been studied only to a very limited exıent in the U.S.A. The work presented in this report is representative of the current American restarch in this area.

Two approaches are being followed in this study of microwave fusion. In one system. operating $i$ a pulsed mode with high-density plasma, the microwave radiation fitelds are used only for heating. and $\mathrm{t} l \mathrm{l}$ : pl:sma core expands inertially. In the other system, using !ow-d'ns:ty plismis. ohe averuge forces arising from the heating fields also confine the plasma during burn. The low-dersity system may have better characteristics for long-term, widespread usi for generating electricity, whereas the high-density systent is somewhit simpler.

The present document. Part 1 of UCRL-51631, treats the interaction of intense microwave radjation and a high-density hydrugen plasma. Part 2, to be published later, will deal with the low-density case. A general discussion of the principles of these microwave fusion systems can be found in Ensley (1974).

For the purposes of this critique. a high-density plasma is one for which tle elcctron tullision frequency exceeds the microwave frequency for $-1 \mathrm{~cm}$ radiation at $-3.5 \mathrm{keV}$ tempersture, and thus the electron density is $210^{20} \mathrm{~cm}^{-3}$. Low-density plasmas are anything with $\mathrm{N}_{\mathrm{e}}<10^{20} \mathrm{~cm}^{-3}$. Also $\mathrm{T}_{\mathrm{e}} \sim \mathrm{T}_{\mathrm{i}}$.

\section{List of Relevant Equations (Gaussian Lirits)}

$$
\begin{gathered}
\nabla \times \vec{E}=-\frac{1}{C} \frac{\partial \vec{B}}{\partial t}, \\
\nabla \times \vec{B}=\frac{4 \pi}{c} \vec{j}+\frac{1}{C} \frac{\partial \vec{E}}{\partial t} . \\
\left.\nabla^{2} \phi=4 \pi \iint f_{e}-f_{i}\right) d \vec{v} . \\
\vec{J}=e \int \vec{v}\left(f_{i}-f_{e}\right) d \vec{v} . \\
\frac{\partial f_{i, e}}{\partial t}+\vec{v} \cdot \frac{\partial f_{i, e}}{\partial r_{j}} \mp-\frac{e}{M_{i, e}}\left[\vec{E}+\frac{\vec{v}}{c} \times \vec{B}+\vec{\nabla}_{\phi}\right]_{j} \cdot \frac{\partial f_{i, e}}{\partial v_{j}}=\left(\frac{\partial r_{i, e}}{\partial t}\right)_{c o l l} .
\end{gathered}
$$




$$
\begin{aligned}
& \sigma=\frac{\mathrm{N}_{\mathrm{e}}(\mathrm{r}) \mathrm{e}^{2} \nu_{\mathrm{e}}}{\mathrm{M}_{\mathrm{e}}\left(\omega^{2}+\nu_{\mathrm{e}}^{2}\right)} . \\
& \nu_{e}=\frac{5 N_{\mathrm{i}} \mathrm{e}^{4} \pi^{3 / 2}}{\left(K T^{3 / 2}\right)^{3 / 2} \mathrm{M}_{\mathrm{e}}^{1 / 2}}, \\
& H_{e}(\tilde{r}, t)=-e \phi(\underline{r} . t)+\frac{(\overrightarrow{\mathbf{P}}+e \vec{A})^{2}}{2 M_{e}}-Z e^{2} \frac{\exp \left[-\gamma \mid \mathbb{R}_{e}(t)-B_{e} \|\right.}{\left|\underline{R}_{e}(t)-\underline{R}_{i}\right|} .
\end{aligned}
$$

Symbols

$\mathrm{H}_{e}=$ electron Hamiltonian with infinite mass ions

$\vec{A}(\underline{g}, t)=$ vector potential of the microwave field

$B_{\mathrm{n}}=$ peak field strength

$\mathrm{P}(\mathrm{t})=$ canonicul momentum

$-\mathrm{e}$ electron charge

$Z e=$ nuclear charge

$\underline{R}_{\mathrm{e}}(t)=$ vector position of electron at time $t$

$\mathbf{R}_{\mathrm{c}}(\mathbf{v})=$ position of ion

$\gamma=1 / \lambda_{\mathrm{D}}=$ cutoff due to Debye shielding

$\lambda_{D}=$ Debye distance

$\sigma=$ risusma conductivity

$T_{i e}=$ ion or electron temperature $\equiv \int \frac{1}{2} M_{i, e} v_{i, e}^{2} f_{i, e} d v$

$v_{\mathrm{i}, \mathrm{e}}=$ ion or electron speed

$N_{i, e}=$ ion or ejectron di sity

$\nu_{e}=$ total elect, on collision frequency

$\delta=$ classical microwave skin depth

$\dot{\phi}=$ high-frequency time average quasi-potential

$\omega_{\text {pi,e }}=$ ion or electron piasma frequency

$E_{b, s}=$ electrs, kinetic energy from bremsstrahlung or transit time

$f_{i, e}=$ ion or electron distribution

$N_{e, i}^{c}=$ plasma density such that $2 N_{e, j}^{t} K T=\frac{\overline{B^{2}+E^{2}}}{8 \pi}$

In general, the solution to Eqs. (1) through (5) must be carried out self-consistently, including $f_{\mathrm{i}, \mathrm{e}}$. As will become apparent later, the calculational problem for low-density plasmas, although solvabl is much more complex than for high-density plasmas. The reason for this is that phase memory of electr as causes surface microwave energy exchange to grossly affect $f_{e}$ for low densities. In systems where a col' sion-dominated core exists, the interior $\mathrm{f}_{\mathrm{e}}$ is nearly independent of the details of particle-field exchanges, except through the effective temperature. 


\section{Plasma-Microwave Interactions}

There are two extensive general categories of interactions between a plasma and microwave field: 1) individual particle behavior, and 2) collective effects, plus interactions between 1) and 2).

All cases considered here will deal with $\omega / \omega_{\mathrm{pe}} \ll 1$. Then $\delta \sim c / \omega_{\mathrm{p}} \ll 1$. Further. $\mathrm{KT}_{e}>1 / 2 \mathrm{keV}$ : also, the radiation wavelength $>0.01 \mathrm{~cm}$. Under these condition $; 5$, the dominant interactions reduce to those discussed below.

\section{INDIVIDI;AL PARTICLE BEHAVIOR}

Stimulated Bremnsstrahlung. The stinulated-bremsstralulung classical cross section is (see Appendix $\mathbf{A}$ ):

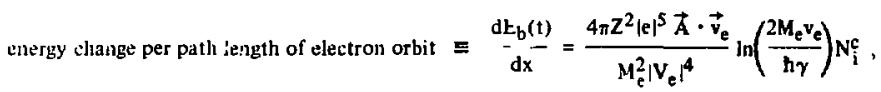

which is valid for arbitrary field strength and spatial dependence, except that:

$$
\frac{\omega}{w_{e}} \cdot \frac{e A}{M v_{e}} \ll 1 \text { and } \frac{1}{|A|}\left|\frac{d A}{d x_{j}}\right| \lambda_{D} \approx 1 \text {. }
$$

and this condition is always met here.

\section{$\underline{\text { Stochastic or Transit-Time Effects }}$}

$$
\Delta E_{s}=-e \int_{J}^{T} \vec{E} \cdot \vec{v}_{e} d t^{\prime}=\left\{\begin{array}{l}
\text { net energy change per "bounce" lasting } \\
\text { time } \tau \text { through the skin field } \vec{E}, \text { per electron. }
\end{array}\right.
$$

In the above equations, it is understood that the fields are self-consistent and the path integrals are taken along the orbits given by $\mathrm{H}_{e}(\mathrm{r}, \mathrm{t})$, Ec. (8), using these fields.

\section{COLLECTIVE EFFECTS}

"Shock" or Collective Collisionless Heating Due to Physicaliy Displacing the Plasma Corona at Twice Field Frequency (see Appendix B). This mechanism arises if the radiation field in the corona is not circularly polarized. Then the lluctuating pressure on the plasma electrons, along the direction of propagation of the field, drives the corona.

Mort work is done by the field when the plasma is "compressed" than the plasma does on the field when it "expands." The heating rate produced, averaged over a period, is:

$$
\dot{\omega}=\frac{1}{T} \int_{0}^{T} P_{f}\left(t^{\prime}\right) V_{p}\left(t^{\prime}\right) d t^{\prime}
$$

where

$$
\begin{aligned}
P_{f}\left(t^{\prime}\right) & =\frac{B_{\lambda}^{2}}{8 \pi} \sin ^{2} \omega t^{\prime}, \\
v_{p} & =\text { corona velocity. } \\
\dot{\omega} & \sim N_{i}^{c} K T\left\langle v_{z}^{i}\right\rangle, \\
\left\langle v_{z}^{i}\right\rangle & \left.=\text { average ion velocity normal to the electrostatic charge wave (along }-\vec{\nabla}_{\phi}\right) .
\end{aligned}
$$




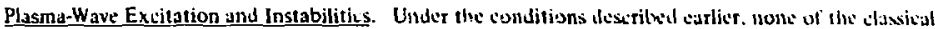
instabilities witich depend for growth on either electron or ion plasma wave resonansts are strongly t'xcitud. Tht reason for this is twofold: First. the spatial region with high-freyuency findi available to sletrons for which the density is reasonably constant is only about ont Debye distunce wide and thus any group of slectrcns only exists under condjicic, 15 where a plasma wave could grow for atout one plusma period. Secondly. the orlirs of any

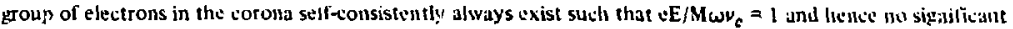
amplification of the two-stream instability coulc takic place.

Another potential mechy "ism is garametricully driven eitetr an and ion glasma oscillutions in tha" drift dircition caused bv the fuctujting held "pump" wase at the houndary. In the low-density system. standing plasmu wave modes might be excited an be driven to larite amplitudes and lied instabilities. This is aroisled if $\omega$ is

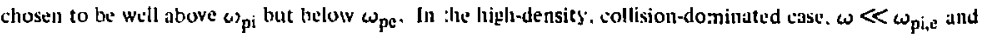
$T_{e}-T_{i}$.

Even so, it may still be that nonline: $r$ effects itre so lapge with very int"nse fiskds in me "pump" microwave

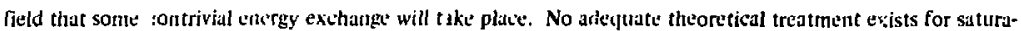
tion under thest conditions. and further theoret sul $n$ ork is needed to understand the saturation of sound waves with $\sim \lambda_{D}$ wavelengths in the plasma.

Hydrorddiation Surface Instabilities. The interface between the external lïeld and plasina is nor in general stable (set Appendix D). Certail plasma surfac: waves niay grow spontaneously. defendia:a upon the initial modes which are excited. If not comteracted with feedback. these may lead to meven disassembly of the plasma. For the low-density system, perfurbations wlitre the surface wavelength oxceds the radiation wavelungth inay grow.

As will be shown, there die some hode conbinations which may exinbit stability to all perturbations, even for long-s:a less than the radiation, and also for which i hydrodynamic disusseinbly time is comparable to moving through a radiation wavelcngth, this is less of a problem.

\section{Hiı̨h-Density Plasma}

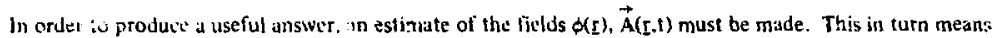
the high-freguency "skin" region of the plisina boundary nust be described. This can be done by solving the self-consistent Maxwell and Pouson cyliations together with the appropriate form of the Liouville equation. Generally, when the skin rution ixtends only over a few interior Debye distances and eneriby exchange rates are so large that even approximate integral invatiants do not exist, it is essential to do numerical self-consistent ricid solutions.

\section{SELF-CONSISTENT FILLISS - HIGH-FREQUENCY SKIN}

In the high-plasma-density cusc, a grtat simplification resules: all phase memory of a partic? lar particle relative to the field is erased by many collisions in crossing the plasma core interior. It is thus really on:. necessiry to solve for the density and current in the lo. -density, "cciiisionless" corona region and join this to the collisional region !: the core as a Maxwellian, as previously mentioned.

The simplest model for conductivity under these conditions is Eqs. (6) and (7).

This canductivity can be somewhat in error due to gross distortions of the electron cloud due to the drift velocity dependence of the Coulomb scuttering cross section (see Appendix A, Part III), but it will be w.sd here to keep tha argument simple. In actual orbit calculations, the collision cross section is used, and the sinple model is used only for rough comparison. 
Next, solve the Hamiltomian fur llac "sollisingless" $\left(w_{\mathrm{e}}<\omega\right)$ repion: his leads in the usual way to the high-

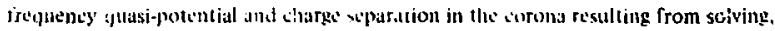

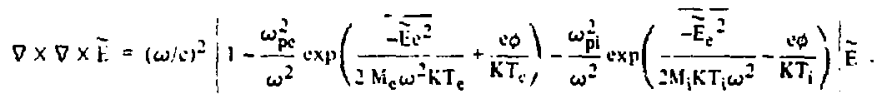

or

$$
\begin{aligned}
& \nabla \times \nabla \times \vec{E}=(\omega / c)^{2}\left[1-\frac{4 \pi L^{2}}{M_{e} \omega^{2}} N_{e}(L)-\frac{4 r^{2}}{M_{i} \omega^{2}} N_{i}(\Sigma)\right\rceil \tilde{E} . \\
& \nabla^{2} \phi=4 \pi q\left[N _ { 1 } \left[[]-N_{\mathrm{c}}[[]) .\right.\right.
\end{aligned}
$$

whicil are Eqs. (1), (2), (3), and (4).

Generally, al though $\nabla^{2} \phi$ exists and there is a considerable difference i $N_{i}$ und $N_{e}$. one can estimate $N_{e}$ alone by making the quasi-neutral approximation: $N_{i}-N_{e}$. This is found numerically to giv the corret order of magnirudo oí $\phi([)$.

$$
\operatorname{co(1)} \simeq\left(\frac{1}{K T_{e}}+\frac{1}{K T_{1}}\right)^{-1} \frac{e^{2} \tilde{\mathrm{E}}^{2}}{2 \omega^{2}}\left(\frac{1}{M_{\mathrm{e}} \mathrm{KT}}-\frac{1}{\mathrm{M}_{\mathrm{i}} \mathrm{KT}}\right) .
$$

and if $T_{i} \sim T_{e}$ and tenoring the term with the ion muss, sou have

$$
\operatorname{co(}(\mathrm{I})=\frac{\overline{\mathrm{e}^{2 \overline{\mathrm{E}}^{2}}}}{4 \mathrm{M}_{\mathrm{s}} \omega^{2}}
$$

and the charge separation field locally is equal to half the quasi-potential at thit point. The quasi-potential itself,

$$
\phi_{\mathrm{e}}=\frac{\overline{\mathrm{e}^{2}} \overline{\mathrm{E}^{2}}}{3 \mathrm{M}_{\mathrm{e}} \omega^{2}} .
$$

is the nonrelativistic result, valid whenever $\phi_{\mathrm{e}}<\mathrm{M}_{\mathrm{e}} \mathrm{L}^{2}$, In stiong fiel, regions. for $\phi_{\mathrm{e}}>\mathrm{M}_{\mathrm{e}} \mathrm{L}^{2}$, this is $\phi_{\mathrm{e}}=\frac{\sqrt{2}}{2} \frac{\mathrm{ce}|\mathrm{E}|}{\omega}$ linear in $\mathrm{E}$ (see Appendix C).

The problem with the above analysis is that ignoring the fluctuations in the electron orbits is equivaknt to guiding center theory. This procedure fails bauly when the time spent by an electron in the find region is iess than a field cycle, so $\omega \tau<1$. This is alway s true if $\left(\omega, \omega_{\mathrm{pe}}\right)<1$ for the case at hand. The next section shows how to replace guiding centes theory for lurge fluctaations in orbits.

\section{ROLE OF FLUCTUATIONS ON THE SKIN DEPTH}

The previous -ilalysis summarizes the theoretical treatment of the average higly-frequency behavior. This 'eads to the classical skin depth $\delta_{\mathrm{c}} \sim c / \omega_{\mathrm{pe}}$. There are, of course, nuctuations. Let us review the reguirements of self-consistency ior $\vec{J}(\mathrm{~L})$ and $\mathrm{N}_{\mathrm{r}}(\mathrm{I})$ corefully. Ignoring ion motion we hed, from Max well's field $\cdots$, ations $\left(\overrightarrow{\mathrm{J}}_{\mathrm{e}}=\right.$ electron current, and $\mathrm{N}_{0}=$ ihterior dersity):

$$
\nabla \times \nabla \times \vec{E}=\left(\frac{\omega}{c}\right)^{2}\left[\vec{E}-\frac{4 \pi}{\omega^{2}} \frac{\partial}{\partial t} \vec{J}_{c}\right] .
$$


and we tried

$$
N_{e}(\Sigma, v)=N_{0}\left(\frac{M}{2 \pi K T}\right)^{3 / 2} \int_{0}^{\infty} \exp \left(\frac{-M v^{2}}{2 K T}-\frac{\overline{e^{2} E^{2}}}{2 M K T \omega^{2}}+\frac{e \phi}{K T}\right) d v
$$

as a soluijon for the electron distribution. The quasi-neutral approximation was

$$
N_{e}(t)=N_{0} \exp \left(\frac{\overrightarrow{e^{2} \vec{E}^{2}}}{4 M_{e} K T \omega^{2}}\right)
$$

and the curtent from $\mathrm{Eq}_{\mathrm{q}}(4)$ is:

$$
\frac{\partial}{\partial t} J_{e}=\frac{e^{2}}{M_{c}} N_{0} \vec{E} \exp \left(\frac{-\overline{e^{2} \vec{E}^{2}}}{4 M \omega^{2} K T_{e}}\right)
$$

Then you have the nonlinear wave equation for $\tilde{E}$, the high-trequency field,

$$
\nabla \times \nabla \times \tilde{E}=\left(\frac{\omega}{c}\right)^{2}\left[1-\frac{\omega_{p e}^{2}}{\omega^{2}} \exp \left(\frac{-\overline{\left.e^{2} \widetilde{E}\right]^{2}}}{4 M \omega^{2} K T_{e}}\right)\right] \underline{\tilde{E}} .
$$

If you now check this equation for self-consistency by summing over a large number of orbit calculations with the Harriltonian using this $\vec{E}$ field and numcrically determine $N(\tilde{c})$ and $\vec{J}(c)$, Maxwell's field equations are not in general satisfjed. The reason is that electrons arriving at one phase of the field have their turning point pulled outward and other phases pushed inward by the fluctuating $\vec{v} \times \vec{B}$ forces, which have no time average. One then iterates with the new $N(r), \vec{J}(r)$ until self-consistency is achieved in Maxwell's equations. This procedure replaces guiding center theory for $\omega_{\text {pe }} / \omega \gg 1$.

In some cases, the quasi-neutral (guiding center) solution is found to reasonably represent the skin-lield behavior, but typically the seli-consistent solutions yield skin depths several times the classical depth. The luctuations thus form the physical limit to the minimum skin depth. The steeper the density gradient from the classical solution, the greater the counteracting fluctuations become.

\section{ROI : GF COLLISIONS ON THE SKIN FIELD}

We have seen that collisionless self-consistent fields can be estimated, e en for very steep density gradients, when fluctuation effects ure important. To see tlie physics involved when $\nu_{e}>\omega$, notice that the orbit from

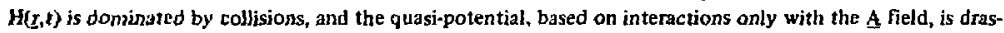
tically reduced, because you now time average orer something close to a random motion rather than a sine wave. The field is then approximately given by

$$
\nabla \times \nabla \times \vec{E}=(\omega / c)^{2}\left[1-\frac{\omega_{p e}^{2}}{\left(\omega^{2}+\nu^{2}\right)}\right] \vec{E},
$$

with collisional skin depth $\delta \sim\left(\frac{\nu}{\omega}\right)^{\not / 2} \frac{\mathrm{c}}{\omega_{\mathrm{pe}}}$. 


\section{SELF CONSISTENT SOLUTIONS}

Choosi the soordilitle system at rest with respect to the average ion velocity. since the high-frequenty skin will form on a time' süle $\tau \sim \delta / v_{j}$; $3 / s o$, it is assumed that the high frequency field relixation time is short compared to liydrodynamic relaxation time.

For convenience in discussion, the quasj-ıeutral e! luation will be used. For thu region $\nu<\omega:$

$$
\nabla \times \nabla \times \underline{E}=\left(\frac{\omega}{c}\right)^{2}\left[1-\frac{\omega_{p e}^{2}-\phi / K T}{\left(\omega^{2}+\nu_{e}^{2}\right)}\right] \underline{E} \text {. }
$$

and $u>\omega:$

$$
\nabla \times \nabla \times \underline{E}=\left(\frac{\omega}{s}\right)^{2}\left[1-\frac{\omega^{2} p e}{\left(\omega^{2}+\nu_{e}^{2}\right)}\right] E: \phi=\frac{\overline{s^{2} E^{2}}}{2 M \omega^{2}} .
$$

Solutions to the first of the above equations are propagating if

$$
\frac{\omega^{2} p e}{\omega^{2}} \mathrm{e}^{-\phi / K T}<1 .
$$

If the field intersity is such that the above inequaliry is reversed at a self-consistent density. $N_{e}$. such that $\nu_{\mathrm{e}}<\omega$ still holds, then the field dies exponentially with $\delta \sim c / \omega p$, and the corona is "collisionless." Fris tlee usual field strengths encountered, $\phi$ is smali and this is the case. However, if the "collisionizss" solution is tried for intense fields suclt that $\nu_{\mathrm{e}}>\boldsymbol{\omega}$, before the ineyuality is reversed, then the nonlinear penetration has delivered ligh-fre fuency field into the collisional regime, and while the first equation holds approximately to the point $\nu_{\mathrm{e}}=\omega$, the field dies exponentially after tizis with

$$
\delta \sim \frac{c}{\omega \cdot J_{p}}\left(\frac{\nu_{e}}{\omega}\right)^{i j}
$$

as it would in a metal.

A further point sliould also be noted: numerical solutions of the nonlinear waye equation always yielu selfconsistent electric field gradients which peak in space near the point where $1 / 2 \mathrm{M}_{\mathrm{e}} v_{\text {th }}^{2} \approx \phi$, the turning point for a thermal electron. In this region the resulting self-consistent peak magnetic field satisfres

$$
\overline{\frac{E^{2}+B^{2}}{8 \pi}}=2 N C K T:\left|B^{2}\right| \gg\left|E^{2}\right| .
$$

Now if you sum the two-componeni Vlasov equation, the well-known result is just tlie plasma pressure balance above. In other words a microwave fjeld under these conditions always nonlinearly penetrates to produce 4 plasma corona at least as dense as that which it coujd collisionlessly confine at the prevailing temperature. This $\mathrm{N}_{\mathrm{e}}^{\mathrm{c}}$ in turn can be large enough so that $\nu_{\mathrm{e}}>\omega$ at say, $3.5 \mathrm{keV}$, through Eq. (7).

\section{APPL! CATION TO HIGH-DENSITY PLASMA HEATING}

Heating rates are estimnted and scaled according to the hydro disassembly time $\tau_{h} \sim r_{0} / v^{1}$ for a sphericul plasma with $r_{0}<\lambda_{R}$. It is shown that, because the quasj-potential energy well for individual particles $\gg K T_{e}$, stochsstic electron heating time potentially can be much shorter than $\tau_{h}$. The wave-particle and skin-cffect heating both have energy doubling times $\sim \tau_{h}$ when $N_{c} / N_{\text {core }} \sim 1$, while the stochastic time $<\tau_{h}$ cven for $10^{-1}<\mathrm{N}_{\mathrm{c}} / \mathrm{N}_{\text {core }}<10^{-2}$. If $\mathrm{N}_{\text {core }} \sim 10^{22}$ and $\mathrm{KT} \sim 3.5 \mathrm{keV}$, then fields of about $>10 \mathrm{MG}$ are needed. These 
in turn may be stored efficiently in a superconducting resonator, probably a pulscd spherical fivity with wall fjelds $\sim 50 \mathrm{kG}$. The dense plasma is then visualized to Q-switul the cavisy by an intenst of bridklown near thi center.

Stimulated [nverse Bremsstrahlung (Skin E[fect). As outlined earlier, with $\nu_{\mathrm{e}} \ \omega$,

$$
v_{z}=-\frac{5 N_{c} e^{4} a^{3 / 2}}{(K T)^{3 / 2} M_{c} 1 / 2} .
$$

where $\omega=$ radistion frequency, with $N_{\mathrm{c}}$ given by

$$
2 \mathrm{~N}_{c} K T_{c}=\frac{\mathrm{B}_{h}^{2}}{3 \pi},
$$

ther in order to stay coupled, with $\omega \sim 10^{11}$ at $3.5 \mathrm{keV}$, requires $\mathrm{N}_{\mathrm{c}} \sim 10^{20}$, so $\mathrm{B}_{\mathrm{n}}>10 \mathrm{MG}$. Under these conditions $\left\{\nu_{\mathrm{e}} \widetilde{\sim} \omega\right)$, the approximate plasma conductivity from the simple model is

$$
\sigma=\frac{N_{\mathrm{c}} \mathrm{e}^{2}}{M v_{\mathrm{e}}}
$$

and the Poynting energy flux into the plasnas sur[ace :s

$$
\begin{gathered}
\overrightarrow{\mathrm{S}}=\left(\frac{B_{\lambda}^{2}}{8 \pi}\right) \cdot\left(\cdot \frac{\left(\nu_{\mathrm{c}} \omega\right)^{1 / 2}}{\omega_{\mathrm{p}}}:\right. \\
\omega_{\mathrm{p}}^{2}=\frac{4 \pi \mathrm{N}_{\mathrm{c}} \mathrm{c}^{2}}{\mathrm{M}}
\end{gathered}
$$

where the collisional skin

$$
\delta=\frac{e}{\omega_{p}} \frac{\left(\nu_{c} \omega\right)^{1 / 2}}{\omega} .
$$

The wel]-known vacuum solutions for tlic eigentields of a spherical resonator are:

$$
\begin{gathered}
\vec{E}=\vec{t} \times \vec{\nabla} \psi . \\
\psi(r, \phi, \theta, t)=e^{i m \phi+i \nu t}\left(A_{e} j_{q}+b_{e} n_{e}\right) P_{e} \cos \theta .
\end{gathered}
$$

and $j_{e}, n_{e}$ are the suherical Besse: and Neumann functions. One property of these solutions is that for $e=1$, a nodal "apple-core" from $\cos \theta$ appears, but also the field near the central conductor is greater than the savity wall by the factor $\sim \frac{2 \pi}{\sqrt{3}} \cdot R / \lambda_{R}$, where $R$ is the cavity radius. Hence a cavity one meter in radius with a $50-k G$ wall field would contuin $\sim 10^{8} \mathrm{~J}$ and with $\lambda_{R} \sim 1 / 4$ to $1 \mathrm{~cm}$. $B_{A}>10 \mathrm{MG}$. The expression for $\vec{S}$ can be written

$$
\vec{S}=P_{\omega} \frac{(2 \pi)^{2}}{3}\left(\frac{R}{\lambda}\right)^{2} \omega \delta,
$$

where $P_{\omega}=$ wall pressure. If you express

$$
P_{\omega} \frac{(2 \pi)^{2}}{3}\left(\frac{R}{\lambda}\right)^{2}=2 N_{c} K T
$$


and compute the enerpy-doubling time $r_{5}$, with $N_{s}=$ ientral densily. then $2 N_{c} K J \omega \delta A \tau_{s}=3 N_{s} K T V$ for a sphere $v i s=r_{0} / 3 . s 0$

$$
\tau_{s}=\frac{1}{2} \cdot \frac{N_{s}}{N_{c}} \frac{r_{0}}{\omega \delta}
$$

su

$$
\tau_{s} / \tau_{h}=\frac{v_{2}}{2(\omega \delta)} \frac{N_{s}}{N_{c}}
$$

hethes $\tau_{s} / T_{1},-1$ requits

$$
\left.N_{\mathrm{c}} / N_{\mathrm{s}} \sim \frac{v_{i}}{2 \omega \delta} \text {, (valid only if } v_{e} \sim \omega\right) \text {. }
$$

Typical values arc $\delta \sim s / \omega_{p} \sim 2 \times 10^{-4} \mathrm{~cm}, \omega \sim 10^{1]}$. so $\mathrm{N}_{\mathrm{c}} / \mathrm{N}_{\mathrm{s}} \sim 1$ for fust hearing. Orbit averages using the bremsstrahlung cross section confirm these heating rutes.

Wave-Particle Heating (See Appendix B). This process hea ts ions. The rate is ${\stackrel{\circ}{\omega_{p}}}_{p}=N_{c} K T\left\langle v^{i}\right\rangle$, where $\left\langle\psi^{j}\right\rangle=$ average radial ion speed. Hence,

$$
\tau_{\omega-p}=r_{0} \frac{N_{s}}{N_{c}} \frac{1}{v_{z}^{i}}
$$

so

$$
\frac{T_{\omega-p}}{T_{h}}=\frac{N_{s}}{N_{c}}
$$

so for fast ion hiejting

$$
N_{c} \sim N_{5}
$$

Stochastic Heating. Stochastic (cransit time) heating can be understood by considering the passage of electrons through a powerful rt field. The rate at which the field does work on an electron entering the field skin region at angle $\theta$ and velocity $v_{\mathfrak{e}}$ when it takes a tinte $z(\phi)$ to exit the field is, per crossing, in the repion just outside the collisional skin,

$$
\Delta E=-\mathrm{e} \int_{0}^{\tau(\phi)} \vec{E} \cdot \vec{V}_{e} d t .
$$

It is understood that all particles are reflected back intu the plasna. Beciuse of the strong field-daminated motjon, the exit time is a function of the phase of the particle relative to the $\vec{E}$ fjeld. This action is analogous to energy exchange in a klystron, except that in this case there is no monoenergetic electron beam with efficient bunching; rather, ihe bunching is small and electrons impinge on the field skin gap from all angles and a Maxwellian speed distribution. Nevertheless, extensive computer studies suniming over the electron dísıribution show strong, net, phase-average heating. Typically for a stochastically tuned system an individual thermal 
electron can be heated by as much as its own energy in one passage in the skin, on the phase average, For this to occur, however, requires the radiation frequency, $\omega$, to approximately satisfy $(\bar{\omega} \bar{\tau}) \sim 10^{-2}$. $\dot{r}=$ averipe transit time $=\delta / \mathrm{ve}_{\mathrm{e}}$. Typical values are, at $3.5 \mathrm{keV}, \delta \sim 2 \times 10^{-4} \mathrm{~cm}, \omega \sim 10^{11}, \mathrm{ve}_{\mathrm{e}} \sim 3 \times 10^{9}$, and $\overline{\omega r} \sim 10^{-?}$. Hellce the desired numerical relationship can $b$ : sproximately sitisfied. The ovtrall hoating rate is then $N_{c} v_{c} K T A$. and the energy doubling time, $\tau_{E}$, is

$$
\boldsymbol{T}_{E}=\frac{2}{3} \mathrm{r}_{0} \frac{\mathrm{N}_{3}}{\mathrm{~N}_{\mathrm{c}}} \frac{1}{v_{\mathrm{e}}}
$$

so for rapid heating,

$$
\frac{\tau_{E}}{\tau_{h}}=\frac{2}{3} \frac{v_{i}}{v_{0}} \frac{N_{s}}{N_{c}}=1
$$

so $\mathrm{N}_{\mathrm{c}} \sim 1 \times 10^{-2} \mathrm{~N}_{5}$, which only requizes one tentil as much $\mathrm{B}$ field as the other mechanioms. The interesting thing about stochastic heating is that it is just the normal, radially inward component of electron energy wlich is increased, and so coupling is very fast.

\section{APPLICATION TO BOOTSTRAP BURN CONDITIONS}

It is well known that to achieve efficient, better than break-even yield in a pulse thermonucleat burn using f: the plasma, say. D-T or D-D, you need to "bootstrap." Since the fusion cross section increasus up to $\sim 100 \mathrm{~kL}$ : you need to couple some of the charged burn products into the ion temperature so the burn rate will exponentiate and require only enough initial cnergy to start the process.

There are scveral methods potentially available to accomplish this objective. Usually, the process involves only Coulomb scattering. For D-T, for instance, the well-known range at, say, solid density, $\sim 2 \times 10^{22}$, is $\lambda_{\alpha} \sim 2 \mathrm{~cm}$ for $\alpha$ particles. Then, in order to have just enough stopping power within the fucl radius, you need a mass $\sim \mathrm{N}_{\mathrm{s}} \mathrm{M}_{\mathrm{i}} \frac{4 \pi}{3}(\lambda \alpha)^{3}$ and an energy supply to an initial fast heating value of, say, $3.5 \mathrm{keV}$ for the ions, which is $\mathrm{E} \sim 3 \mathrm{~N}_{\mathbf{s}} \mathrm{KT}$ volume and $\lambda \alpha \sim 1 / \mathrm{N}$. Hence the usual relationship for required energy at a given temperature is $\mathrm{E} \sim 1000\left(\frac{\mathrm{N}_{5}}{\mathrm{~N}}\right)^{2}$ in megajoules. If one is somc what limited in available energy, then there is an obvious advantage to increasing $\mathrm{N}$.

There is another way the bootstrap condition can be achieved with much smaller $E$ and $N \sim N_{s}$. Instead of relying entirely on Coulomb scattering in a "one-shot" process, th charged bum products can be effectively coupled to the matter if they are trapped at the surface electrostatically. The charge-separation ficld at the boundary which confines ions also will reflect, for instance, any charged burn product with energy $<20 \mathrm{MeV}$.

In the case of $\alpha$ particles, for instance, $v_{\alpha} / v_{\mathrm{i}} \sim 100$, so an $\alpha$ particle can make about 100 reverberations per $\tau_{h}$. The initial energy goes with the physical : lass, so you now require $r_{d} \sim \frac{v_{i}}{v_{\alpha}} \lambda \alpha$ :

$$
E-3 N_{s} K T \frac{4}{3} \pi \lambda \alpha^{3}\left(v_{i} / v_{\alpha}\right)^{3}
$$

or $E \sim\left(\frac{N_{s}}{N}\right)^{2}$ in kilojoules.

We hive seen that because of the quasi-potential for electrons a powerful charge-separation electric field arises and can confine individual charged burn products. Another logical question is the collective behavior. The average hot $\alpha$ component, for instance, is 


$$
N_{\alpha}=\frac{N_{i}^{2}}{4} \overline{\sigma v} \frac{\lambda_{\alpha}}{v_{\alpha}} .
$$

where $\lambda_{\sigma} / r_{\alpha}$ is the mean alplua lifistine. Since the energy is $-3.5 \mathrm{MeV}$, the momentum flux into the chargescparation fictu will equal the cold ions at $N_{\alpha}=10^{18}$. snec we are assuming the conditions for $\mathrm{KT}_{\mathrm{e}} \sim 10 \mathrm{keV}$ and $N_{c}-10^{20}$. Thus the diploa particles are electrastically coupled to the ion core because in order to pull collectively the clectrons through the field they need a density $\mathrm{N}_{\mathrm{e}} \sim \mathrm{N}_{\mathrm{c}}$ lor pressure balance. and this would imply a large density excess $\left(N_{c} / N_{\alpha} \sim 10^{2}\right)$ for the electron whicle would then be bound to the core. Furclier numeritul studies with the tluse-component equation coupled through Poisson's equation wre being undertuken to try to better understand the collective behavior of the tharged burn products. including wave-particle coupling.

At present it would ippear that under the conditions outlined above. Jefinite bootstrup to about $10 \mathrm{keV}$ in D-T is expected, in solid density ( $\bar{\sigma}_{\mathrm{D}} \mathrm{D}-\mathrm{T}-10^{-16} \mathrm{at} 10 \mathrm{ke}$ ). Then the $\alpha$ 's are expected to retmain coupled cullectively to the (ion $+a$ ) core hydroexpansion speed in order to preserve over-ill charge neutrality, and tontimue the process until burn is mostly over. Of course. such small drops miglu disassemhle too fast for large fractional burn-up if $\mathrm{N}$ does not increase.

It is illteresting to consider the subject of very-high-efticiency busn resulting from strong burn coupled wave particle interaction which will be considered as a portion of a forthcoming seport on inethods of direct conversion. 


\section{Appendix .1 \\ STIMULATED BREMSSTRAHLUNG}

\section{A CLASSICAL DERIVATION OF STIMUL,ATED BREMSSTRAHLUNG}

Consider the motion of an electron past an infinitely heavy stationary ion of charge $\mathrm{Ze}$, while in a circularly polarized standing wave radiation field. The Hamiltonian of the electron is:

$$
H=\frac{\overrightarrow{\mathbf{P}}(t)-\mathrm{q} \overrightarrow{\mathrm{A}}(\mathrm{z}, \mathrm{t})\}}{2 \mathrm{M}_{e}}-\frac{Z \mathrm{e}^{2} \exp \left[-\gamma\left|\vec{R}_{e}(t)-\vec{R}_{c}\right|\right]}{\left|\vec{R}_{e}(t)-\vec{R}_{c}\right|},
$$

where

$\vec{P}(t)$ is the canonical momentum of the electron,

$q$ is the electron charge, equal to $-\mathrm{e}=-4.803 \times 10^{-10} \mathrm{esu}$,

$\vec{A}(z, t)$ is the vector potential, which I take equal to $\vec{A}(z, t)=A(z)(\sin \omega t, \cos \omega t .0)$,

$A(z)=$ self-consistent field,

$\vec{R}_{e}(t)$ is the vector position of the electron at time $t$,

$\vec{R}_{c}$ is the position of the ion, $\vec{R}_{c}=\left(X_{c}, Y_{c}, Z_{c}\right)$,

$\gamma$ is an exponential cutoff due to Debye shielding $\gamma=\frac{1}{\lambda_{D}}$.

I will henceforth consider the problem of $A(z)=A$, a constant at any point in the skin. If it were not for the small perturbation of the particle orbit due to the jon, the solution of Hamilton's equations would be

$$
\begin{aligned}
& R_{0}(t) \equiv\left[x_{0}(t), y_{0}(t), z_{0}(t)\right], \\
& x_{0}(t)=x+\frac{q A}{M_{e} \omega}\left(\cos \omega t-\cos \omega t_{0}\right)+\frac{P_{x_{0}}\left(t-t_{0}\right)}{M_{e}} . \\
& y_{0}(t)=y-\frac{q A}{M_{e} \omega}\left(\sin \omega t-s_{1}: \omega t_{0}\right)+\frac{P_{y_{0}}\left(t-t_{0}\right)}{M_{e}}, \\
& z_{0}(t)=z+\frac{P_{z 0}\left(t-t_{0}\right)}{M_{e}},
\end{aligned}
$$

where $(x, y, z)$ is the position of the particle at the initial time $t_{0}$ and $\frac{\vec{P}_{0}}{M_{e}}-\frac{q \vec{A}\left(t_{0}\right)}{M_{c}}$ is the velocity at this initial time.

Choose $t_{0}$ to be the timie tue unperturbed particle passes through that plane through the ion such that the veloc:ty of the particle is perpendicular to the plane (Fig. .41). 


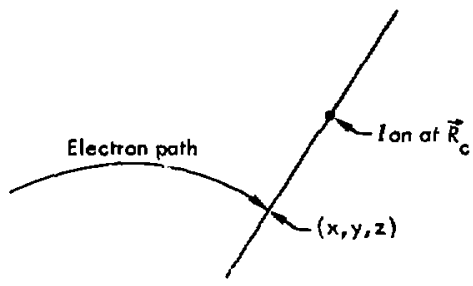

Fig. Al.

This point $\vec{R} \equiv(x, y, z)$ is the point of closist approach of ilte unperturbed particle, and very near the point of closest approuch of the perturbed particle. For the derivation to be unambiguously followed. the dield strength. field frequency, electron velocity, and plasma parameters must be such that there is only one such point $\vec{R}$ within the Debye sphere of the ion, but this is hardly a restrictive assumption.

To solve for the motion of the particle in the electromagnetic field and Coulomb field, treat the Coulomb field as a perturbation and use the following jreration loop.

Using the unperturbed position $\vec{R}_{0}(t)$ and canonical momentum $\vec{P}_{0}$, and the full Hamiltonian of p. 12 on the right-hand side (only), write down and solve (by integrating explicity witl respect to timli) Hamilton's equations

$$
\begin{aligned}
& \frac{d x_{i}}{d t}=\frac{\partial H}{\partial \dot{F}_{i}}, \\
& \frac{d P_{i}}{d t}=-\frac{\partial H}{x_{i}},
\end{aligned}
$$

for the first-order position and canonical momentum. Plug these first-order values into the right-hand side of Hamilton's equations to obtain the second-order values, etc. Repeat as many times as it takes to obtain thr desired accuracy.

The first-order position vector is

$$
\begin{aligned}
\vec{R}_{1}(t)= & \vec{R}_{0}(t)-\frac{Z e^{2}}{M_{e}} \int_{-\infty}^{t} d t^{\prime} \int_{-\infty}^{t^{\prime}} d t^{\prime \prime} \frac{\left[\vec{R}_{0}\left(t^{\prime \prime}\right)-\vec{R}_{c}\right] \exp \left[-\gamma\left|\vec{R}_{0}\left(t^{\prime \prime}\right)-\vec{R}_{c}\right|\right]}{\left|\vec{R}_{0}\left(t^{\prime \prime}\right)-\vec{R}_{c}\right|^{3}} \\
& \frac{-\gamma \mathrm{Ze}^{2}}{M_{e}} \int_{-\infty}^{t} d t^{\prime} \int_{-\infty}^{t^{\prime}} d t^{* \prime} \frac{\left[\vec{R}_{0}\left(t^{\prime \prime}\right)-\vec{R}_{c}\right] \exp \left[-\gamma\left|\vec{R}_{0}\left(t^{\prime \prime}\right)-\vec{R}_{c}\right|\right]}{\left|\vec{R}_{0}\left(t^{\prime \prime}\right)-\vec{R}_{c}\right|^{2}} .
\end{aligned}
$$

It turns out to be sufticient for present purposes to have the second-order expression for the time derivative of the canonical momentum:

$$
\frac{d \vec{P}}{d t}=\frac{-Z e^{2}\left[\vec{R}_{1}(t)-\vec{R}_{c}\right] \exp \left[-\gamma\left|\vec{R}_{1}(t)-\vec{R}_{c}\right|\right]}{\left|\vec{R}_{1}(t)-\vec{R}_{c}\right|^{3}}-\frac{\gamma e^{2}\left[\vec{R}_{1}(t)-\vec{R}_{c}\right] \exp \left[-\gamma\left|\vec{R}_{1}(t)-\vec{R}_{c}\right|\right]}{\left|\vec{R}_{1}(t)-\vec{R}_{c}\right|^{2}} .
$$

Just how big is the perturbation in the position of the particle? The minimum impact parameter the electron can have is $R_{\min }=\frac{h}{M_{e} v} \mathrm{~cm}$. (This condition clearly comes from quantum mechanics - witness the $h$ - so in this way, the calculation isn't really classical. For a justification of this minimum impact parameter. see. for examnle. 
Jackson, Classical Electrodynamics, sections 13.3 and 15.2). The maximum deflection tl:: partitle cun make from its unperturbed path is (impulse approximation)

$$
=\frac{Z e^{2}}{M_{e} R_{\min }^{2}} \Delta T^{2}
$$

where $\Delta \mathrm{T}$ is the interaction time. $\Delta \mathrm{T}=\frac{\mathrm{R}_{\mathrm{mn}}}{\mathrm{v}}$.

This deflection as a percentage of the minimum impact parameter is

$$
\frac{Z_{\mathrm{e}^{2}}}{\mathrm{M}_{\mathrm{e}} \mathrm{v}^{2}} \frac{\mathrm{M}_{\mathrm{e}}^{\mathrm{v}}}{\mathrm{h}}=\frac{\mathrm{Ze}^{2}}{\mathrm{hv}}=\frac{2.5 Z \times 10^{\mathrm{g}}}{\mathrm{v}}
$$

This supplies a pretty lax condition on $\frac{Z}{v}$ for the previous and following approximaticas to be valid:

$$
2.5 \times 10^{8} \frac{Z}{v} \ll 1
$$

Having shown that the terms of $\vec{R}_{1}$ (t) proportional to $Z \mathrm{e}^{2}$ are much smaller than $\vec{R}_{0}(t)$. expand the expression if $\frac{\mathrm{dP}}{\mathrm{dt}}$, droppine terms of higher thar, second order in $\left(\mathrm{Ze}^{2}\right)$.

Define

$$
\begin{aligned}
& \vec{a}\left(Z \mathrm{e}^{2}\right)=\frac{Z \mathrm{e}^{2}}{\mathrm{M}_{\mathrm{e}}} \int_{-\infty}^{\mathrm{t}} \mathrm{dt^{ \prime }} \int_{-\infty}^{\mathrm{t}^{\prime}} \mathrm{dt^{ \prime \prime }} \frac{\left[\overrightarrow{\mathrm{R}}_{0}\left(\mathrm{t}^{\prime \prime}\right)-\overrightarrow{\mathbf{R}}_{c}\right] \operatorname{s\sim }\left[-\gamma\left|\overrightarrow{\mathrm{R}}_{0}\left(\mathrm{t}^{\prime \prime}\right)-\overrightarrow{\mathbf{R}}_{\mathrm{c}}\right|\right]}{\left|\overrightarrow{\mathrm{R}}_{0}\left(\mathrm{t}^{\prime \prime}\right)-\overrightarrow{\mathrm{R}}_{\mathrm{c}}\right|^{3}} . \\
& \vec{B}\left(Z e^{2}\right)=\frac{Z e^{2} \gamma}{M_{e}} \int_{-\infty}^{t} d t^{\prime} \int_{-\infty}^{t^{\prime}} d t^{\prime \prime} \frac{\left[\vec{R}_{0}\left(t^{\prime \prime}\right)-\vec{R}_{c}\right] \exp \left[-\gamma\left|\vec{R}_{0}\left(t^{\prime \prime}\right)-\vec{R}_{c}\right|\right]}{\left|\vec{R}_{0}\left(t^{\prime \prime}\right)-\vec{R}_{e}\right|^{2}} \text {, } \\
& \left.\frac{d \vec{P}}{d t}=-Z e^{2} \exp \left[-\gamma \mid \vec{R}_{0}(t)-\vec{R}_{c}\right)\right]\left[\vec{R}_{0}(t)-\vec{R}_{c}\right]\left[\vec{R}_{0}(t)-\vec{R}_{c}\right] \cdot\left[\vec{a}^{\prime}\left(Z e^{2}\right)+\not \vec{\beta}\left(c e^{2}\right)\right] \\
& \cdot\left[\frac{3}{\left|\vec{R}_{0}(t)-\vec{R}_{c}\right|^{5}}+\frac{3 \gamma}{\left|\vec{R}_{0}(t)-\vec{R}_{c}\right|^{4}}+\frac{\gamma^{2}}{\left|\vec{P}_{0}(t)-\vec{R}_{c}\right|^{3}}\right] \\
& +Z^{2} \exp \left[-\gamma\left|\vec{R}_{0}(t)-\vec{R}_{t}\right|\right]\left[\vec{\alpha}\left(Z^{2}\right)-\vec{\beta}\left(Z e^{2}\right)\right]\left[\frac{1}{\left|\vec{R}_{0}(t)-\vec{R}_{c}\right|^{3}}+\frac{\gamma}{\left|\vec{R}_{0}(t)-\vec{R}_{c}\right|^{2}}\right] \\
& -Z^{2} \exp \left[-\gamma\left|\vec{R}_{0}(t)-\vec{R}_{c}\right|\right]\left[\vec{R}_{0}(t)-\vec{R}_{c}\right]\left[\frac{1}{\left|\vec{R}_{0}(t)-\vec{R}_{c}\right|^{3}}+\frac{\gamma}{\left|\vec{R}_{0}(t)-\vec{R}_{c}\right|^{2}}\right] .
\end{aligned}
$$

Now, make a pair of further, somewhat related assumptions. First, assume that the field period of the radit tion is very long compared to the inferaction time of the electron with the ion. This amounts to $\frac{\omega}{\gamma^{v}} \ll 1$.

Alternately, this can be rewritten (where $\lambda$ is the wavelength of the radiation) $2 \pi \frac{\lambda_{D}}{\lambda} \frac{c}{v} \ll 1$.

For electron velocities high enough to :atisfy the previous assumptions and plasmas of densities of interest, this condition is easily met. 
The explicit meaning of $t / 2$ second asstumption can be seen by notine that $\vec{R}_{1}(t)$ can be rewritten as

$$
\begin{aligned}
\vec{R}_{1}(t)= & x+\left(\frac{P_{x 0}}{M_{e}}-\frac{e A}{M_{e}} \sin \omega t_{0}\right)\left(t-t_{0}\right)+\left[\frac{e A}{M_{e} \omega}\left(\cos \omega t-\cos \omega t_{j}\right)+\frac{e A}{M_{e}} \sin \omega t_{0}\left(t-t_{0}\right)\right], \\
& y+\left(\frac{P_{y 0}}{M_{e}}-\frac{e A}{M_{e}} \cos \omega t_{0}\right)\left(t-t_{0}\right)+\left[\frac{e A}{M_{e} \omega}\left(\sin \omega t_{0}-\sin \omega t\right)+\frac{e A}{M_{e}} \cos \omega t_{0}\left(t-t_{0}\right)\right], \\
& \left.7+\frac{P_{z 0}\left(t-t_{0}\right)}{M_{c}}\right\} .
\end{aligned}
$$

Define $\quad \vec{v} \equiv\left(\frac{P_{x 0}}{M_{e}}-\frac{e A}{M_{e}} \sin \omega t_{0}, \frac{P_{y 0}}{M_{e}}-\frac{e A}{M_{e}} \cos \omega t_{0}, \frac{P_{z 0}}{M_{e}}\right)$.

$$
\vec{R}(t) \equiv \vec{R}-\vec{R}_{t}+\vec{v}\left(t-t_{0}\right)
$$

$\vec{v}$ is just the velocity of the (unperturbed) electron at the position $\vec{R}$,

$$
\vec{v} \cdot\left(\vec{R}-\vec{R}_{e}\right)=0 \text {. }
$$

and therefore,

$$
|\vec{R}(t)|^{2}=\left|\vec{R}-\vec{R}_{c}\right|^{2}+v^{2}\left(t-t_{0}\right)
$$

Since $\vec{E}(t)=-\frac{\partial \vec{A}(t)}{\partial t}$,

$$
\vec{R}_{0}(t)-\vec{R}_{c}=\vec{R}(t)+\frac{e}{M_{i}} \overrightarrow{A_{1}}\left(t_{0}\right)\left(t-t_{0}\right)+\frac{e \vec{E}\left(t_{0}\right)}{M_{e} \omega^{2}}-\frac{e \vec{E}(t)}{M_{q} \omega^{2}}
$$

The exact value of $\vec{R}_{0}(t)-\vec{R}_{c}$ is important only when its magnitude is less than several Debye lengths. I have already assumed that the field turns very little while the electron is in this region. The field function can thus be accurately expanded around $t_{0}$ :

$$
\begin{aligned}
\vec{R}_{0}(t)-\vec{R}_{c} & =\vec{R}(t)+\frac{e}{M_{e}} \vec{A}\left(t_{0}\right)\left(t-t_{0}\right)+\frac{e \vec{E}\left(t_{0}\right)}{M_{e} \omega^{2}}-\frac{e \vec{E}(t)}{M_{e} \omega^{2}} \\
& =\frac{e \vec{E}\left(t_{0}\right)\left(t-t_{0}\right)^{2}}{2 M_{e}}+\frac{e \vec{A}\left(t_{0}\right) \omega^{2}\left(t-t_{0}\right)^{3}}{6 M_{e}}
\end{aligned}
$$

This piece of $\vec{R}_{0}(t)-\vec{R}_{c}$ represents the deviation from straight-line motion of the electron in the vicinity of the jon. I henceforth assume this deviation to be small while the particle is interacting with the ion. The maximum relevant value of $\left|t-t_{0}\right|$ is $\frac{1}{\gamma v}$, so this assumption is equivalent to $\frac{e A \omega}{M_{e} \gamma^{2} y^{2}} \ll \frac{1}{\gamma}$, or $\frac{\omega}{\gamma v} \frac{e A}{M_{e} v} \ll 1$.

Note that this is the only rastriction on the strength of the field made in this derivation. Thus, the result will be of much wider validity than that found, for example, by the standard perturbation methods of quantum electrodynamics. The field can be strong enough to completely turn the particle around, as long as the field frequency is low enough so that the particle is not deflected a sizeable fraction of a Debye length while traveling across a Debye sphere. 
It remains to simplify the expression for $\frac{d \vec{p}}{d t}$ and use it to evaluate the energy cluange of the electron. As this is merely a mathematical procedure with litcle further pliysicul content. it will be britt.

Every where in the expression for $\frac{d \vec{p}}{d t}$, expand

$$
\left|\vec{R}_{0}(t)-\vec{R}_{c}\right|^{2}=|\vec{R}(t)|\left[1+\frac{\vec{R}(t) \cdot\left[\frac{e \vec{E}\left(t_{0}\right)\left(t-t_{0}\right)^{2}}{2 M_{e}}+\frac{e \vec{A}\left(t_{0}\right) w^{2}\left(t-t_{0}\right)^{3}}{6 M_{e}}\right]}{|\vec{R}(t)|^{2}}\right] .
$$

It turns out. though I won't demonstrate it here, that the

$$
\frac{\vec{R}(t) \cdot\left[\frac{\vec{E}\left(t-t_{0}\right)^{2}}{2 M_{e}}+\frac{e \vec{A}\left(t_{0}\right) \omega^{2}\left(t-t_{0}\right)^{3}}{6 M_{e}}\right]}{|\vec{R}(t)|^{2}}
$$

piece leads to a contribution to the energy change of the electron which is down by at least

$$
\frac{\omega}{\gamma v} \frac{e A}{M_{e} v}\left[\ln \left(\frac{2 M_{e} v}{h \gamma}\right)\right]^{-1}
$$

from other contributions. Therefore I drop this piece now, and the expression for $\frac{d \vec{P}}{d t}$ on $p$. 14 is valid with $\vec{R}_{0}(t)-\vec{R}_{c}$ replaced every where by $\vec{R}(t)$. The time average energy of the unperturbed electron is a constant. Therefore the energy change due to stattering irom the ion is

$$
\Delta E=\int_{-\infty}^{\infty} \frac{d H}{d t} d t .
$$

Since the electron motion is a solution of Hamilton's equations, accurate to sufficient order for present needs, $\frac{\partial H}{\partial t}=\frac{j H}{d t}$.

$$
\begin{aligned}
\Delta E & =\int_{-\infty}^{\infty} d t \frac{\partial}{\partial t}\left\{\frac{\mid \vec{P}-e \vec{A}(t)]^{2}}{2 M_{e}}\right\} \\
& =\frac{-A \omega}{M_{e}} \int_{-\infty}^{\infty} d t\left[\left(P_{x} \cos \omega t\right)-\left(P_{y} \sin \omega t\right)\right] .
\end{aligned}
$$

Integrate by parts, ignoring the term evaluated at the limits as having time average zero.

$$
\Delta E=+\frac{q}{M_{e}} \int_{-\infty}^{\infty} \text { dt } \vec{A}(t) \cdot \frac{d \vec{P}}{d t}
$$

where $\frac{d \overrightarrow{\mathrm{p}}}{\mathrm{dt}}$ is given by the p. 14 expression.

Actually, one is interested in the energy change of the electron as it moves by all the ions down its path. Therefore, integrate over all the ions in a plane perpendicular to the velocity $v$ at a given time, and then later integrate along the path of the electson to get the conitibstion of all the ions in the plasma. Rely once more on 
thx "small bending combition." dure lave injact parameters muth larger than $\frac{1}{\gamma}$, and hence are of litlle significance (see Fig. A2).

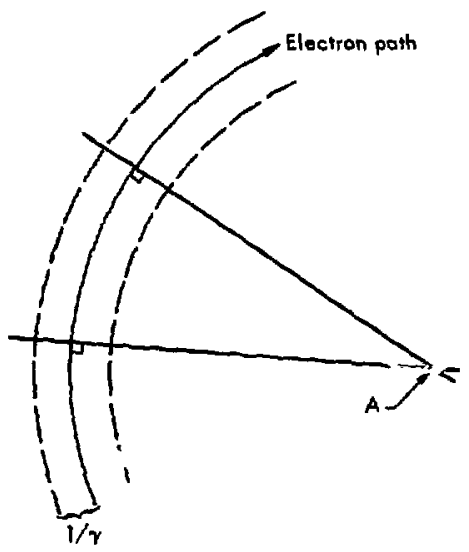

Fig. A2. Only the ions between rhe docted lines matier much, so the fast that the tons at point A get double-counted is unimportant.

$$
\frac{\Delta \mathrm{E}}{\Delta \mathrm{x}}=N_{\mathrm{f}} \int d \vec{R}_{\mathrm{c}}\left[\frac{\mathrm{q}}{\mathrm{M}_{e}} \int_{-\infty}^{\infty} \mathrm{dt} \overrightarrow{\mathrm{A}}(t) \cdot \frac{\mathrm{d} \overrightarrow{\mathrm{P}}}{\mathrm{dt}}\right],
$$

where the $d \vec{R}_{\mathbf{L}}$ integral is over $\left|\vec{R}-\vec{R}_{c}\right|>R_{\text {min }}$.

$$
\begin{aligned}
& \Delta E=N_{i} \int d s \frac{d E}{d s}, \\
& N_{i}=\text { ion density } .
\end{aligned}
$$

and the integral os is along the path of the electron.

The integril over impact parameters eliminates the lineas in $2 \mathrm{e}^{2}$ terms of $\frac{\mathrm{d} \overrightarrow{\mathrm{P}}}{\mathrm{dt}}$. as they are antisymmetric with respect to the impact parameter. (Or rather the resultant piece of $\Delta E$ is lintearly proportional to $\mathbf{R}_{\min }$ and small. Physically speaking, this term is proportional to the average field of the uniform-density ions. which is zero, rather than the rms field of the uniform density jons, which is not. See, for example, Boyd and Sanderson, Plasma Dymamics, pp. 234-235.)

Derine tile symbol

\begin{tabular}{ll}
$\frac{N}{2}$ & $\frac{M}{2}$ \\
\hline
\end{tabular}

$$
A^{3} \mid K L
$$


to mear

$$
A \int_{R_{\min }}^{\infty} R^{J+1} d R \int_{-\infty}^{\infty} \frac{d t t^{K} \exp \left[-\gamma\left(R^{2}+v^{2} t^{2}\right)^{1 / 2}\right]}{\left(R^{2}+v^{2} t^{2}\right)^{N / 2}} \int_{-\infty}^{t} \frac{d t^{\prime} t^{\circ L} \exp \left[-\gamma\left(R^{2}+v^{2} t^{2}\right)^{1 / 2}\right]}{\left(R^{2}+v^{2} t^{2}\right)^{M / 2}} .
$$

The expression for $\frac{\Delta E}{\Delta x}$ above becomes. upon plugging in the expression for $\frac{d \vec{P}}{d t}$ on $p .14$ and doing the $d t$. integra, by parts:

$$
\frac{\Delta \mathrm{E}}{\Delta x}=\frac{2 \pi \mathrm{q}}{\mathrm{M}_{\mathrm{c}}} \frac{\left(\mathrm{Ze}^{2}\right)^{2}}{\mathrm{M}_{\mathrm{E}}} \mathrm{N}_{\mathrm{i}}
$$

' . . Tes terms listed below. Terms which are proportional to $\vec{A} \cdot \vec{R}$ and $\vec{E} \cdot \vec{R}$ have been dropped as antisymmetric in the impact parameter.

\begin{tabular}{|c|c|c|c|c|c|c|c|}
\hline$\frac{2}{2}, \frac{2}{2}$ & $\frac{2}{2}, \frac{3}{2}$ & $\frac{3}{2}, \frac{2}{2}$ & $\frac{3}{2}, \frac{3}{2}$ & $\frac{4}{2}, \frac{2}{2}$ & $\frac{4}{2}, \frac{3}{2}$ & $\frac{5}{2}, \frac{2}{2}$ & $\frac{5}{2}, \frac{3}{2}$ \\
\hline$-t^{2} \mid 11$ & $+\gamma \mid 2 z$ & $+\gamma \mid 11$ & $+\mid 1\}$ & $-3 \gamma^{2} R^{2} 120$ & $-3 \gamma \mathbf{R}^{2} 120$ & $-3 \gamma R^{2}[20$ & $-3 R^{2} 120$ \\
\hline$-r^{2} 102$ & -102 & $-\gamma \mid 02$ & -102 & $+3 \gamma^{2} R^{2} \| 1$ & $+3 \gamma R^{2} \mid 11$ & $+3 \gamma R^{2}\{1\}$ & $+3 R^{2} \mid \| 1$ \\
\hline & & $-\gamma^{3} R^{2} \mid 20$ & $-\gamma^{2} R^{2} \mid 20$ & $-3 \gamma^{2} v^{2}|3|$ & $-3 \gamma^{2}|3|$ & $-3 r^{2}|3|$ & $-3 v^{2} \mid 31$ \\
\hline & & $-\gamma^{3} \mathbf{R}^{2}|\||$ & $+\gamma^{2} R^{2} \mid 11$ & $+3 \gamma^{2} v^{2} \mid 22$ & $+3 \gamma^{2} 122$ & $+3 r^{2} 122$ & $+3 v^{2} \mid 22$ \\
\hline & & & & & & & \\
\hline & & & & & & & \\
\hline
\end{tabular}

$\vec{A} \cdot \vec{v}$

$\overrightarrow{\mathrm{E}} \cdot \overrightarrow{\mathrm{v}}$

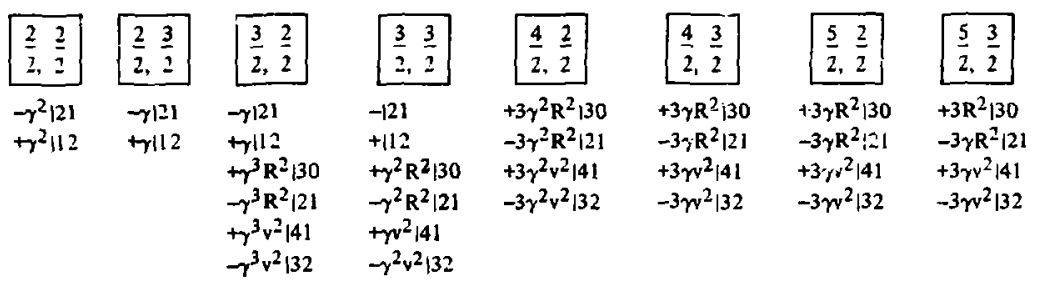

The $\vec{E} \cdot \vec{v}$ terms turn out to lead to contributions which are $\approx \frac{1}{25} \frac{\omega}{\gamma}$ times the magnitude of the leading $\vec{A} \cdot \vec{v}$ term. The final result is (ignoring terms with the same angular dependence and the same coefficient without the logariutati):

$$
\frac{d E}{d x}=\frac{4 \pi Z^{2} \mid e^{5}}{M_{e}^{2} v^{4}} \vec{A} \cdot \vec{v} \ln \left(\frac{2 M_{e} v}{h \gamma}\right) N_{i}
$$




\section{Il. RELATION TO THE QUANTUM TREATMENT OF SSIMULATED BREMSST RAHLUNG}

Stimulated $\mu \mathrm{r}$ gessis are usually thought of as being inherently quanfum effects. In the case of low-frequency

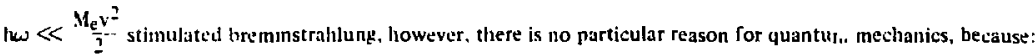

1. There are no bound states.

2. Intesaction with a single photon does not change the momentum or energy of the electron by a significant fraction of the initial values.

3. The photon field in problems of interest is clearly strong enough to be treated as an externally inposed classical hisld (i.c.. changing the photon population by a fiew photons has no effect on the problem)

4. The wave nature of the purticles is unimportant other than in determining the minimum classical impact parameter of the electron, and this is sasily fudged into ste classical salculation.

The stamedard quantum-mechanical treatment of a problem of this type approximates the elstron wave function by a plane wave. Thus, to recover the quantum result, it is necessary to construct the classical analogue of a plane $v$ ave.

Consider an ion imbedded in a hall space of uniform $\vec{A}=A\left[\sin \omega^{*}, \cos \omega^{+}, 0\right] f_{i} \cdot\left(u^{2}\right.$. with the surface of the half-space perpendicular to the $\vec{\nabla}$ of interest. Rain ions with vrlocity, $\vec{v}_{0}$, random inp.ct purameters, and random times of arrival at the field edge down on the surface of the half plane (Fig. A3):
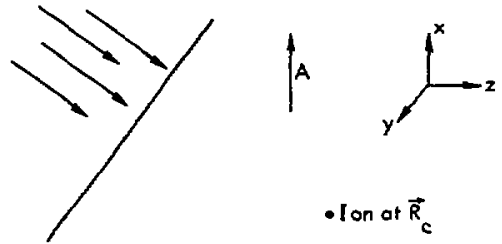

\section{- Ion at $\vec{R}_{\mathrm{c}}$}

Fig. A3.

$$
v=v_{0}(\sin \theta \cos \phi \cdot \sin \theta \sin \phi \cdot \cos \theta) .
$$

Perform a coordinate rotation to put the velocity along the $z$ axis:

$$
\vec{v}=v_{0}\left[\begin{array}{ccc}
\cos \theta & 0 & -\sin \theta \\
0 & 1 & 0 \\
\sin \theta & \cos \theta
\end{array}\right]\left[\begin{array}{ccc}
\cos \phi \sin \phi & 0 \\
-\sin \phi & \cos \phi & 0 \\
0 & 0 & 1
\end{array}\right]\left[\begin{array}{ll}
\sin \theta & \cos \phi \\
\sin \theta & \cos \phi \\
\cos \theta
\end{array}\right]=v_{0}\left[\begin{array}{l}
0 \\
0 \\
1
\end{array}\right] .
$$

The $\overrightarrow{\mathbf{A}}$ field is now:

$$
\overrightarrow{\mathbf{A}}(1)=A\left[\begin{array}{l}
\sin \omega t \cos \phi \cos \partial+\sin \phi \cos \theta \cos \omega t \\
-\sin \phi \sin \omega t+\cos \phi \cos \omega t \\
\sin \theta \cos \phi \cos \omega t+\sin \theta \sin \phi \cos \omega t
\end{array}\right] .
$$


Let $z_{E}$ be the $z$ coordinate of the field edge, and $t_{E}$ the time of arrival at the field edge. Treating every thing from now on to lowest order in the field, obtain:

Outside the field:

$$
\left.\begin{array}{l}
x(t)=x_{0} \\
y(t)=y_{0} \\
z(t)=z_{E}+v_{0}\left(t-t_{E}\right)
\end{array}\right\} t<t_{E}
$$

Inside the field:

$$
\vec{R}(t)=\{x(t), y(t), z(t) !
$$

where

$$
\begin{aligned}
& x(t)=\frac{-q A}{M_{e} \omega}\left[-\left(\cos \omega t-\cos \omega t_{E}\right) \cos \phi \cos \theta+\left(\sin \omega t-\sin \omega t_{E}\right) \sin \phi \cos \theta\right]+x_{0}, \\
& y(t)=\frac{-q A}{M_{e} \omega}\left\{+\sin \phi\left(\cos \omega t-\cos \omega t_{E}\right\}+\cos \phi\left(\sin \omega t-\sin \omega t_{E}\right)\right]+y_{0}, \\
& \left.z(t)=2_{E}+v_{0}\left(t-t_{E}\right)-\frac{q A}{M_{e} \omega} I-\left(\cos \omega t-\cos \omega t_{E}\right) \sin \theta \cos \phi+\left(\sin \omega t-\sin \omega t_{E}\right) \sin \theta \sin \phi\right] .
\end{aligned}
$$

The zero-order time of closest approach is

$$
T=t_{E}+\frac{\left(z_{c}-z_{E}\right)}{v} .
$$

The first-crder time of closest approach $T_{1}$ can be found from

$$
\left.\mid \vec{R}(T)-\vec{R}_{c}\right\rceil \cdot \vec{v}_{0}(T)=0
$$

This yields $T_{1}=T+\Delta T$, where

$$
\begin{aligned}
\Delta T= & \frac{q A}{M_{e} v_{0}^{2}}\left\{\frac{v_{0}}{\omega} f-\left(\cos \omega T-\cos \omega t_{E}\right\} \sin \theta \cos \phi+\left(\sin \omega T-\sin \omega t_{E}\right) \sin \theta \sin \phi\right\} \\
& +\left(x_{0}-x_{c}\right)(\sin \omega T \cos \phi \cos \theta+\sin \phi \cos \theta \cos \omega t) \\
& \left.+\left(y_{0}-y_{c}\right)(\sin \phi \sin \omega T+\cos \phi \cos \omega T)\right\}
\end{aligned}
$$

The impact-parameter-dependent pieces of $\Delta T$ turn out not to affect the result. (It is not necessary to go back and do all the impact-parameter integrals to show that this is true. Examine the time average of the impact terms where $\Delta \mathrm{T}$ is used below.) 
Use lliesc results to swaluate the energy shange of the electron:

$$
\begin{aligned}
\frac{d E}{d s} & =\frac{4 \pi Z^{2} e^{5}}{M_{e}{ }^{2} v^{4}} \vec{A}\left(T_{1}\right) \cdot \vec{v} \ln \left(\frac{2 M_{e} v}{h \gamma}\right) N_{i} \\
& =\frac{\left.4 \pi Z^{2} v^{5}\left[\vec{A}(t)+\frac{\Delta T \partial \vec{A}}{\partial T}\right] \cdot \mid \overrightarrow{v_{0}}+\vec{E}\right]}{M_{e}{ }^{2}|\vec{v}+\vec{e}|^{4}} \ln \left(\frac{2 M_{e}|\vec{v}+\vec{E}|}{h \gamma}\right) N_{i} .
\end{aligned}
$$

where

$$
\vec{\epsilon}=\frac{-\mathrm{qA}}{\mathrm{M}_{\mathrm{e}}}\left[\begin{array}{l}
\sin \omega \mathrm{T} \cos \phi \cos \theta+\sin \phi \cos \theta \cos \omega \mathrm{T} \\
\sin \phi \sin \omega \mathrm{T}+\cos \phi \cos \omega \mathrm{T} \\
\sin \theta \cos \phi \sin \omega \mathrm{T}+\sin \theta \sin \phi \cos \omega \mathrm{T}
\end{array}\right] .
$$

The expression for $\Delta E$ to first order in $\vec{A}$ is zero when averaged over impact times. Average the various second order in $\vec{A}$ pieces over impact times:

$$
\begin{aligned}
& \langle\vec{A} \cdot \vec{e}\rangle=\frac{-|| A^{2}}{2 M_{e}}\left[\cos ^{2} \phi \cos ^{2} \theta+\sin ^{2} \theta \cos ^{2} \phi+\sin ^{2} \phi \cos ^{2} \theta+\sin ^{2} \phi+\cos ^{2} \phi+\sin ^{2} \theta \sin ^{2} \phi\right]=\frac{-q A^{2}}{M_{e}} \\
& \frac{1}{\vec{v}_{0}+\left.\epsilon\right|^{4}}=\frac{1}{v_{0}^{4}}\left(1-\frac{4 \vec{\epsilon} \cdot \vec{v}_{0}}{v_{0}^{2}}\right) \text {. } \\
& \left\langle\vec{A} \cdot \vec{v}_{0} \vec{\epsilon} \cdot \vec{v}_{0}\right\rangle=\frac{-q A^{2} v_{0}^{2}}{2 M_{e}}\left(\sin ^{2} \theta \cos ^{2} \phi+\sin ^{2} \theta \sin ^{2} \phi\right)=\frac{-q A^{2} v_{0}^{2} \sin \theta}{2 M_{e}} \text {. } \\
& \left\langle\Delta T \frac{\partial \vec{A}\left(T_{0}\right)}{\partial T_{0}} \cdot \ddot{v_{0}}\right\rangle=\frac{-g A^{2}}{2 M_{\mathrm{e}}}\left(\sin ^{2} \theta \cos ^{2} \phi+\sin ^{2} \theta \sin ^{2} \phi\right)=\frac{-q \mathrm{~A}^{2} \sin ^{2} \theta}{2 M_{\mathrm{e}}} . \\
& \ln \left(\frac{2 \mathrm{M}_{\mathrm{e}}|\overrightarrow{\mathrm{v}}+\vec{\epsilon}|}{h \gamma}\right)=\ln \left(\frac{2 \mathrm{M}_{\mathrm{e}} \mathrm{v}_{0}}{\mathrm{~h} \gamma}\right)+\frac{\overrightarrow{\boldsymbol{\epsilon}} \cdot \vec{v}_{0}}{v_{0}^{2}} .
\end{aligned}
$$

yields no non-zero averaged log terns.

Putting all the pieces together,

$$
\begin{aligned}
& \frac{\partial E}{\partial x}=\frac{4 \pi Z^{2} \mathrm{e}^{5} \ln \left(\frac{2 M_{\mathrm{e}} v}{\ln }\right)}{M_{\mathrm{e}}^{2} v^{4}}\left(\frac{+q A^{2}}{M_{e}}\right)\left(-1+2 \sin ^{2} \theta-\frac{\sin ^{2} \theta}{2}\right) N_{i}, \\
& \frac{\partial E}{\partial x}=\frac{-2 \pi Z^{2} e^{6} A^{2}}{M_{e}{ }^{3} v^{4}} \ln \left(\frac{2 M_{e} v}{\pi \gamma}\right)\left(3 \sin ^{2} \theta-2\right) N_{i} .
\end{aligned}
$$

where $q=\_$. This is, finally, the dominant term of the quantum result in the $\omega / \gamma^{v} \ll 1$ case.*

*D. Marcuse, Bell Sy'stem Tech. J. 4J, 1557 (1962) and 42, 415 (1963). Note that his result is for a linearly polanized A field. Putting in a circularly' polarized fiuld yields the above result. 


\section{COLLISIONAL MICROWAVE SKIN HEATING}

We are dealing with a self-consistent plasma-field bouvlary region. This represents an equilibrium between i fully ionized hydrogen plasma and a circularly polarized microwave hickl. We waut to salculite tlic rate at which the ordered, field-driven motion of the electrons, in thi: "skin" field region, is transformed into heat due to ion collision when the thermal motion of the electrons is considered.

The approach of equivalent fields will be used. (See Trubnikov for a general theoretical treatment. especially pp. $175-181$ ).

1. Average Momentum Exchange Betwuen Electrons and Ions. Trubnikov noticed that, for a Coulomb intıraction. the mean velocity change for a test particle, $\alpha$. moving through a cloud of field particles, $\beta$, could be written as

$$
\left\langle\Delta v_{i}\right\rangle^{\alpha / \beta}=\left(1+\frac{m_{a}}{m_{\beta}}\right) \lambda\left(4 \pi e_{\alpha} e_{\beta} / m_{a}\right)^{2} \frac{\partial}{\partial v_{i}}\left(\frac{i}{4 \pi} \int f_{\alpha} \frac{\left(\underline{y}^{\prime}\right) d \underline{\underline{y}}^{\prime}}{\mid \underline{\underline{v}-\underline{v}^{\prime} \mid}}\right) .
$$

by explojting the [act that the form of this function is mathematically identical in velocity space to the problem in ordinary spave of finding the electrostatic field at an observation point due to an arbitrary "source" distribution of charge at points, with $\underline{r}^{\prime} \rightarrow \underline{v^{\prime}}$. Tha force of dynamic friction slong the test particle velocity can thus be written

$$
\vec{F}_{i}=\frac{d \vec{P}_{\alpha}}{d t}=-q_{\alpha}\left(1+\frac{m_{\alpha}}{m_{\beta}}\right) \overrightarrow{\mathrm{E}}_{\alpha}
$$

where

$$
E_{\alpha}=-\nabla \phi_{\alpha},
$$

and

$$
\phi_{\alpha}=\int \frac{f_{\beta\left(v^{\prime}\right) d \underline{v}^{\prime}}}{\mid \underline{v}-\underline{v^{\prime} \mid}} .
$$

Now by analogy with electrostatics, the $\mathrm{F}_{-\alpha}$ can be written down for any distribution $f_{\beta}\left(v^{\prime}\right)$. From Gauss's theorem, any spherica!ly isotropic velocity distribution will produce an equivalent "fieis" at field point $\underline{y}$ of

$$
E_{\underline{v}}^{\alpha}=\frac{4 \pi \int_{0}^{\underline{v}} f_{\rho}\left(\underline{v}^{\prime}\right) d \underline{v}^{\prime}}{4 \pi \underline{v}^{2}} ; \vec{F}_{1}:-\left(\frac{\vec{v}_{\alpha}}{\left|\vec{v}_{\alpha}\right|}\right) q_{\alpha}\left(1+\frac{m_{\alpha}}{m_{\beta}}\right) E_{\underline{v}}^{\alpha} .
$$

2. Heating and Comparison with Stimulated Bremsstrahlung. Using the simple "moving Maxwellian" model for electrons in the s'sin, normalized to $n_{\beta}$,

$$
\int_{0}^{|\vec{v}(E)|} f_{\beta}\left(v^{\prime}\right) d v^{\prime}=n_{\beta} \frac{4}{\sqrt{\pi}} \int_{r}^{|\vec{v}|} e^{-\nu_{\beta}^{2}} j_{\beta}^{2} d \nu_{0} .
$$

where we have transformed to the coordinate frame at rest with respect to the average electron velocity $\vec{v}(E)$ and $|\vec{v}|=|\vec{v}(E)|\left(\frac{m_{\beta}}{2 K T e}\right)^{3 / 2}$. 
$\vec{v}(E)$ is a solution using local self-consistent hinds $\vec{E}$ of the equation of motion.

$$
M_{e} \overrightarrow{\vec{v}}(E)=-c \vec{E}+\nu_{e} M_{e} \vec{v}(E)
$$

delining

$$
\mu\left(\nu_{i}^{2}\right)=\frac{2}{\sqrt{\pi}} \int_{0}^{v_{i}^{2}} \mathrm{e}^{-\eta} \sqrt{\eta} \mathrm{d} \eta .
$$

The "lest particle" force, or, in the present case, the mean force on an ion is. $n_{j} \cong N_{E}: m_{i p} \equiv M_{e}$ :

$$
F_{i}=-\hat{v}_{i} \varphi_{i}\left(l+\frac{M_{i}}{M_{c}}\right) \frac{N_{e} M_{c}}{2 K T_{e} \nu_{i}^{2}} \cdot \mu\left(\nu_{i}^{2}\right) \hat{\nu}_{i} \equiv \frac{\vec{r}(E)}{|\vec{v}(E)|} .
$$

where

$$
q_{i} \equiv 4 \pi \lambda \frac{e_{i}^{2} e_{\beta}^{2}}{M_{i}}
$$

is the effective "charge" of the test particle (jon). Hence, one lias the heating rate $\vec{F}_{i} \cdot \vec{v}(E) N_{i}$, which, after dropping unity conpared to $M_{i} / M_{e}$, is

$$
\dot{\omega}=\frac{N_{i}\left([ ) N _ { e } \left([) 4 \pi \lambda z^{2} e^{4}\right.\right.}{\left(2 K T_{e} M_{e}\right)^{3 / 2}} \frac{\mu\left(\nu^{2}\right)}{\nu_{i}} .
$$

When orbit calculations are run for stimulated bremsstrahlung in tine microwave skin, a vilue near the sbove is obtained. Further if $\nu_{e}>\omega$, the usual "skin effect" heating rate for a metal applies, and the value obtained from it,

$$
\dot{\omega}_{\text {skin etrect }}=\frac{B_{i}^{2}}{8 \pi} C \cdot\left(\frac{\nu_{e} \omega}{\omega_{P_{e}}}\right)^{1 / 5} .
$$

is alsa compurabt: to the other two, so that in this case,

$$
\dot{\omega}_{\text {skin effect }}=\dot{\omega}_{\text {collisions }} \approx \dot{\omega}_{\text {stim. bremsstrahlüII }} \text {. }
$$




\section{Appendix B \\ COLLECTIVE COLLISIONLESS ION HEATING}

Under conditions of interest, $\nu_{e} / \omega_{p e i} \ll 1$. and effects of interpurticle tollisions on orbits can be ignored while particles are turning around in the skin region. We wish to calculate the eneroy exchange between the time dependent $\mathrm{A}^{2}$ potential and the plasm. Since the corons satisfies the conditions for self-consistent confinement on the average, the pressure fuctuations are at least $\sim 2$ NSKT and cannot be considet $i$ a small perturbation. Average hydrodynamic motion is igtoned uver one cycte of the ligh-frequency field. Here $N_{i}$ satjsfies

$$
\frac{\overrightarrow{B^{2}+E^{2}}}{8 \pi}=2 N_{i} K T
$$

First of all, to simplify the discussion, consider radii of curvature, physical dimensions, etc., to corsspond to a plane radiation front. Secondly, we are considering plane polarized radiation since the effect vanishes for a perfectly circular $A$ field. $\left(A^{2}(t)=\right.$ constant in time, then.) Hence take $\vec{A} \times \hat{x} A(2)$ sin $\omega t$. For most problems of interes, $\omega<\omega_{\text {pej. }}$ Since we are considering seff-consistent solutions, we will work with $B^{2} / 8 \pi$ mather than $A^{2}$.

$$
\frac{\sqrt{B^{2}+E^{2}}}{8 \pi} \equiv P_{f}=\text { field pressure exeried on the skin electrons. }
$$

When $P_{f}$ tises fium zero, the electron pressure, which is in equiliorium with the field, has two components: one kinetic and one electrostatic due to the ions. (See Fig. BL.)

$$
\begin{aligned}
& \eta=\text { relative coordinate betwcen electrons and ions. } \\
& \equiv \frac{1}{N_{i}^{c}} f\left(N_{e}-N_{i}\right) d z=\text { average turning point for electrons relative } \\
& z_{p}=\text { inertial coordingte of the average classical turning point for ions. } \\
& i_{p} \equiv v_{p} \text {. } \\
& \dot{\eta}=\frac{d n}{d t} \text {. } \\
& \text { eq, }=\text { ion wecterating force, } \\
& Q^{2}{ }_{\phi}=4 \pi e \int\left(f_{e}-r_{i}\right) d V_{i} \text {. } \\
& N_{e j}=\int \mathrm{e}_{e, j} d v_{e, j} \text {. }
\end{aligned}
$$

The electrostatic component depends upon $n$; the kinetic component depends upon $\dot{\eta}_{\mathbf{v}} v_{\mathbf{p}}$.

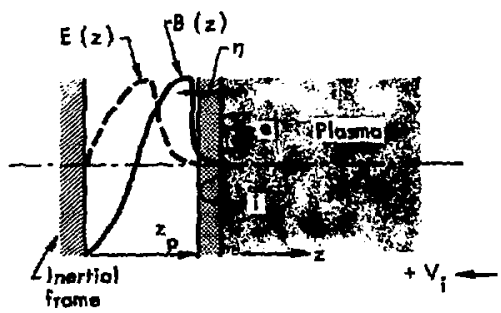

Fit 81. 
Since $2 \omega \ll \omega_{\text {pj }}$, the ions will follow the phase of the acceleroting field generated by $\eta$. There is a purely elcctrostatic potential associated with o for which the field energy stared by the $P_{r}$ is given back to $P_{f}$ every cycle. The irfeversible work rote kinetioully per cycte is

We now compute $V_{P}\left(t^{\prime}\right)$.

$$
\dot{W}=\frac{-1}{\tau} \int_{0}^{\tau}\left(P_{f}^{j} V_{p}+P_{f}^{e} \dot{\eta}\right) d t^{\prime}=\frac{-l}{\tau} \int_{0}^{T} P_{f}\left(t^{\prime}\right) V_{p}\left(t^{\prime}\right) d t^{\prime}
$$

From the Lorentz force equation, you must have continuity of mechanical and electromagnetic forces at each phase of the clectromagnetic field

$$
\begin{aligned}
& P_{f}(t)=2 N_{i}^{c} M_{i} \int_{V_{z}^{i}=V_{n}^{z} \text { if } V_{n}^{z}>0}^{i} V_{j}^{i}\left(V_{z}^{i}-V_{P}^{z}\right)^{2} d V_{z}^{i} \\
& =0 \text { if } V_{\mathrm{p}}^{2}<0 \\
& +2 N_{e}^{c} M_{e} \int_{v_{z}^{e}=\left(\dot{\eta}+v_{p}^{2}\right) i r\left(\dot{\eta}+v_{p}^{z}\right)>0}^{\infty} f_{e} v_{z}^{e}\left[v_{z}^{e}-\left(\dot{\eta}+v_{p}^{z}\right)\right]^{2} d v_{z}^{e} \\
& =0 \text { if }\left(\bar{\eta}+V_{p}^{2}\right)<0
\end{aligned}
$$

This equation comes about because the flux of particles $v^{e, j}$ in to a moving wall is $\alpha\left(v_{l}^{e, 1}-v_{p}^{2}\right.$ ) (inertial irame)

$V_{p}^{z}=$ velocity of the wall, and the corresponding elastic change in momentum is $\alpha\left(V_{i, e}^{z}-V_{p}^{z}\right)$ per particle, where $V_{e, i}^{2}$ is the initial velocity of the particles and hence $>0$ only. but $V_{p}^{2}$ is $\leq 0$. We are simply calculating the self-consistent pressure against a moving self-consistent electrostatic "wall."

First, it is shown that $\dot{n}\left\langle V_{e}\right\rangle$ is negligible. We know that

$$
e_{\max } \sim\left(\frac{e^{2} A^{2}}{2 M}\right)_{\max }=4 K T
$$

hence,

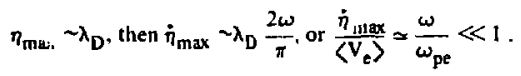

Expanding the tight-hand side (RHS) of the above expression. leaving wit the superscripts on $N_{c, i}$ where it is understood from now on that these refer to average electron and ion densities corresponding to the confinement relation (the lower integration limits for $V_{z}^{i}$ and $V_{z}^{e}$ are as given above):

$$
\begin{aligned}
R H S= & 2 N_{i} M_{j} \int^{\infty} f_{i}\left(V_{z}^{i}\right)^{2} d V_{z}^{i}-4 N_{i} M_{i} V_{p}^{z} \int^{\infty} f_{j} V_{z}^{i} d V_{z}^{i} \\
& +2 N_{j} M_{i}\left(V_{p}^{2}\right)^{2} \int^{\infty} f_{i} d V_{z}^{i} \\
& +2 N_{e} M_{e} \int^{\infty} f_{e}\left(V_{z}^{e}\right)^{2} d V_{z}^{e}-4 N_{e} M_{e}\left(\dot{\eta}+V_{P}^{z}\right) \int^{\infty} f_{e} V_{z}^{e} d V_{z}^{e} \\
& +2 N_{e} M_{e}\left(V_{p}^{z}+\dot{\eta}\right)^{2} \int^{\infty} f_{e} d V_{z}^{e} .
\end{aligned}
$$

As stated earlier, the above are the initiai distribution functions. Butause of phase memory loss in crossing the system, the initial distribution function of a displaced Maxwellian is the distribution at any time.

Taking note of the above magnitudes,

$$
\text { RHS }=2 N_{i} M_{1}\left\langle\left(V_{z}^{i}\right)^{2}\right\rangle+2 N_{e} M_{e}\left\langle\left(V_{z}^{e}\right)^{2}\right\rangle+N_{i} M_{i}\left(V_{p}^{z}\right)^{2}-4 N_{i} M_{i} V_{p}^{z}\left\langle v_{2}^{i}\right\rangle
$$


where

$$
\begin{aligned}
\left\langle v_{z}^{i}\right\rangle=\sqrt{\frac{K T}{2 \pi M_{i}}} & \\
\left\langle\left(v_{2}^{i}\right)^{2}\right\rangle & =\frac{K T}{2 M_{i}} .
\end{aligned}
$$

so

$$
\begin{aligned}
& \overline{P_{C}\left(t^{\prime}\right)}=2 N_{i} K T+2 N_{i} M_{i} \overline{\left(V_{p}^{2}\right)^{2}} \text { since } \int_{0}^{2 \pi} V_{p}^{2}\left(t^{\prime}\right) d t=0 . \\
& \frac{B_{A}^{T}}{16 \pi}=2 N_{i} K T+N_{i} M_{i} \overline{\left(V_{p}^{2}\right)^{2}} . B_{\Lambda}=\text { peak field strenght. }
\end{aligned}
$$

For the time-dependent motion, rewrite

$$
\begin{aligned}
& P_{(}\left(t^{\prime}\right)=2 N_{i} M_{i}\left(\frac{M_{i}}{2 \pi K T}\right)^{I / 2} \int_{z}=0 \text { ir } v_{p}^{z}>0 \\
&=-v_{p}^{z} \text { If } v_{p}^{z}<0
\end{aligned}
$$

$V_{p}^{z}<0$ means $z_{p}$ is imireasing, a "compnession, "whicin means $P_{f}(t)>\bar{P}_{f}$ and $\frac{\pi}{4}<\omega t<\frac{3 \pi}{4}$.

The solution to the above equation for this phase range is

$$
v_{p}\left(t^{\prime}\right)=2\left\langle v_{2}^{i}\right\rangle(1-\sqrt{1-\pi \cos 2 \omega t}) \text {. }
$$

so $\left|V_{p}\right|$ luals a negative peak $\sim 3\left\langle v_{z}\right\rangle$. $V_{p}>0$ : "expansion" with $P_{f}(t)<\overrightarrow{P_{f}}$

$$
\begin{gathered}
0<\omega t^{\prime}<\frac{\pi}{4} \text { and } \frac{3}{4 \pi}<\omega t<\pi \\
P_{f}\left(t^{\prime}\right)=2 N_{j} M_{i}\left(\frac{M_{i}}{2 \pi K T}\right)^{1 / 2} \int_{0}^{\infty} \exp \left[\frac{-M_{i}}{2 K T_{i}}\left(z+V_{p}^{t}\right)^{2}\right] z^{2} d z .
\end{gathered}
$$

which must be evaluated numerically. However, when $\omega t=0, \pi_{\text {, we must have } V_{p}} \sqrt{\frac{K T}{M_{i}}}$, since this tornesponds to no field pressure and "Free" expansion. This part of the cycle does not contribute much work back on the field. however, in any case, because $P_{f} \sim 0$. A sketch of $\dot{W} . P_{f}\left(t^{\prime}\right), v_{p}\left(t^{\prime}\right)$ is shown in Fig. $B$ ?.

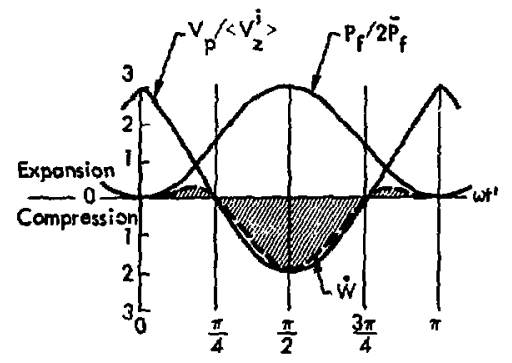

Fig. B2. 


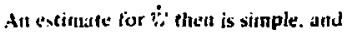

$$
\dot{W}-\left\langle v_{z}^{i}\right\rangle=\frac{\pi}{\omega} \frac{B 2}{B \pi} \int_{\pi / 4 \omega}^{\pi i 2 \omega} \sin 2 \omega t^{\prime}\left(1-\sqrt{1-\frac{\pi}{2} \cos \omega t^{\prime}}\right) d t^{\prime}
$$

Using a iriangle trom $\frac{\pi}{4}$ iv $\frac{\pi}{2}$, you have

$$
\dot{W}-\frac{4 \omega}{\pi} \frac{B_{n}^{2}}{8 \pi} \frac{\pi}{16 \omega}\left\langle V_{z}^{i}\right\rangle=\frac{B_{\lambda}^{2}}{8 \pi}\left\langle\frac{\left.V_{z}^{i}\right\rangle}{4}=\frac{4 N_{i} K T_{1}}{4}\left\langle v_{z}^{i}\right\rangle .\right.
$$

so

$$
\dot{\mathbf{w}}=\mathbf{N K T}\left\langle\mathbf{v}_{\mathbf{z}}^{\mathbf{i}}\right\rangle \text {. }
$$

This represents the work done directly on the ions due 10 ion kinetic friction.

The above calculation can also be looked upon as a wave-particle interaction in which a strong time-dependent field produces an electrostatic wave which couples to the particles, when you have trapping from one direction.

The above method is used because with large nonlinear behavior and steep field and density pradients in the skin, a purely formal mathematical solution to the coupled Vlasov-Maxweli-Poisson set is unknown.

It should also be borne in mind that any periodic motion of the surface of a hot plasma satisfying the confinement (and other) conditions always requires irreversible expendirure of energy.

Generalizution to other geometry eigenfields $\vec{A}(\underline{r}, t)$ and arbitrary polarization is straightforward. 


\section{Appendix C \\ RELATIVISTIC QUASI-POTENTIALS}

Ignoring collisions, the relativistic Hamiltonian for an electron is

$$
H e=e \phi+c\left[\left(m_{0} c\right)^{2}+p^{2}+e^{2} A^{2}+2 e(\vec{P} \cdot \vec{A})\right]^{1 / 2} .
$$

Writing $m v_{\perp}=P_{1}+e A_{\perp}$ and choosing $\vec{A}=\vec{A}_{y}(z)+\vec{A}_{x}(z)$ for plane radiation.

$$
\begin{aligned}
P_{L} & \equiv P_{x}+P_{y}, \\
A_{\perp} & \equiv A_{x}+A_{y} . \\
(1-\beta) & \equiv 1-(\dot{z} j c)^{2} . \\
i & =\text { spesd along field gradient } . \\
\alpha & \equiv \frac{\left.\mid P_{\perp}^{2}-2 e P_{x} A_{x}-2 e P_{y} A_{y}+e^{2} A_{1}^{2}\right]}{\left(m_{0} c\right)^{2}} .
\end{aligned}
$$

Then you can write

$$
-\dot{\mathrm{P}}_{2}=\frac{\partial H}{\partial z}=\mathrm{e} \frac{\partial \phi}{\partial z}+\frac{m_{0} c^{2}}{2}\left(\frac{1-\beta}{1+\alpha}\right)^{1 / 2} \frac{\partial \alpha}{\partial z} .
$$

For $\alpha \gg 1, \beta \ll 1$.

$$
|\alpha|=\frac{e^{2} A^{2}}{2 m_{0}^{2} e^{2}}, \quad \sqrt{\alpha}=\frac{e A}{\sqrt{2} m_{0} c},
$$

so

$$
-F_{2}= \pm e \frac{\partial \phi}{\partial z}+\frac{\sqrt{2}}{4} c e \cdot \frac{1}{A} \frac{\partial}{\partial z} A^{2}
$$

or

$$
-F_{z}=\frac{\partial}{\partial z}\left(\operatorname{te\phi } \frac{\sqrt{2}}{2} \frac{\mathrm{ceE}}{\omega}\right)
$$

So the quasi-potential is, for $|\vec{v}| \rightarrow c$,

$$
\bar{\phi}=\frac{\sqrt{2}}{2} \frac{\mathrm{eE}}{\omega} \text {. }
$$

It might be noted that you car: write

$$
\mathbf{m}=\mathrm{m}_{0} \cdot\left(\frac{1+\alpha}{1-\beta}\right)^{\frac{1}{2}},
$$


and it should be shown that in unis formulation $\vec{v} \cdot \vec{v}<c^{2}$. since we have field-driven motion that is valid for arbitrary field strengtl. Let $P_{1}=0$ : tlierefore,

$$
v_{\perp}=\frac{e A L}{m_{0}}\left(\frac{1-\beta}{1+\alpha}\right)^{1 / 2}=\left(\frac{e E}{m_{0} \omega}\right)\left(\frac{1-\beta}{1+\alpha}\right)^{1 / 2} \text {. }
$$

so

$$
\left|v_{1}\right|^{2}=\left(\frac{\mathrm{eE}}{\mathrm{m}_{0} \omega}\right)^{2} \frac{1}{(1+\alpha)}-\left(-\frac{\mathrm{eE}}{\mathrm{m}_{0} \omega}\right)^{2} \frac{1}{(1+\alpha)} \frac{\dot{i}^{2}}{\mathrm{c}^{2}} .
$$

so

$$
v_{1}^{2}+\left(\frac{e E}{m_{0} \omega}\right)^{2} \frac{1}{(1+\alpha)} \frac{\dot{z}^{2}}{c^{2}}=\left(\frac{e E}{m_{0} \omega}\right)^{2} \frac{1}{1+\alpha} .
$$

For very intense fields, $(1+\alpha) \rightarrow \alpha$; hence, in the limit of infinitely strong fields,

$$
v_{1}^{2}+\dot{z}^{2}=c^{2}
$$




\section{Appendix D}

\section{PLASMA HYDRORADIATION STABILITY}

Solutions to self-consistent field equations generally assume shape stability for the plasma-radiation eigenfield interface. We now consider the question of stability for such an interface. To simplify the discussion. a planar geometry will be assumed, with no loss in generality.

The general method will involve the basic assumption that the microwave filld relaxes much lister than the plasma surface, so that any hydrodynamic motion of the plasna as a whole cun be innored in somputing the fields.

Perturbation theory can be used to evaluate changes in the field intensity resulting from any particular Fourier surface-wave component. The resulting fonces either tend to restore the surface to the original uniformity ("stable") or re-enforce the perturbation ("unsta sle").

\section{FIELD PERTURBATION}

A surface shape $\zeta=\zeta_{0} \cos k_{z} z$ is considered at $\tau=0$.

The plasma surface normal is:

$$
\hat{n}_{s}=-\sqrt{\frac{\eta^{2}-1}{\eta^{4}-1}}(\eta \hat{x}+\hat{z})
$$

where

$$
\eta=1 / \zeta_{0} k_{z} \sin k_{z} z \text {. }
$$

For $\eta \gg 1$,

$$
\hat{n}_{5}--\left(\hat{x}+\hat{z} 5_{0} k_{2} \sin k_{2} z\right) .
$$

Separation of the wave equation gives for the perturbation field $E^{\prime}$ (or $B^{\prime}$ )

$$
E^{\prime}\left(B^{\prime}\right)=g^{\prime} h^{\prime} e^{i \omega t} \text {. }
$$

(The surface wavelength $\lambda_{z}=2 \pi \mathrm{k}_{z}{ }^{-1}$ need not be considered smaller than the collisionless skin depth, because in that case the skin acts as a dielectric lens and washes out the assumed perturbations.)

$$
\frac{d^{2} g^{\prime}}{d z^{2}}+k_{z}^{2} g^{\prime}(z)=0, \frac{d^{2} h^{\prime}}{d x^{2}}+h^{\prime}\left(k_{0}{ }^{2}-k_{z}{ }^{2}\right)=0,
$$

with solutions:

$$
\gamma=\sqrt{k_{0}^{2}-k_{z}^{2}},
$$

$\gamma^{2}>0: E_{i}^{\prime}=\left(A_{i} \sin \gamma x+B_{i} \cos \gamma x\right) e^{i k_{2} z} e^{i \omega t}$.

$\gamma^{2}<0: E_{i}=\left(A_{i} \sinh \gamma x+B_{i} \cosh \gamma x\right) e^{i k_{2} z} e^{i \omega t}$.

$k_{0}=$ original wavenumber of the field without the perturbation. 
We now do a first-order perturbation in which the eigenfrequency remains essentially unchanged. Treating the plasma is a perfict conductor as well as the walt, the fields must satisfy

$$
\begin{aligned}
& \nabla \cdot \vec{B}=0 \text { interior } \\
& \nabla \cdot \vec{E}=0 \text { interior charge-free region } \\
& \left.\begin{array}{r}
\hat{n}_{s} \cdot \vec{B}=0 \\
\left.\frac{\partial B}{\partial \hat{n}}\right|_{s}=0 \\
\times \vec{E})=0
\end{array}\right\} \text { at surficte } \\
& \overrightarrow{\mathrm{F}}=\overrightarrow{\mathrm{E}}^{0}+\overrightarrow{\mathrm{E}}^{\prime} \\
& \vec{B}=\vec{B}^{0}+\vec{B}^{\prime} \text {. }
\end{aligned}
$$

$\mathrm{E}^{0}, \mathrm{~B}^{\mathrm{D}}$ are the eigenfields with no perturbation. Wi first consider the case where the surface troughs lie along $E^{0}$. So the zero fields are

$$
\begin{aligned}
E_{y}^{0} & =\hat{y} B_{0} \sin \left(\frac{n \pi}{l}\right) x, \\
B_{z}^{0} & =\hat{z} B_{0} \cos \left(\frac{n \pi}{q}\right) x, \\
B_{z}^{\prime} & =(A \sin \gamma x+B \cos \gamma x) e^{i k_{z}^{z} e^{i \omega t},} \\
B_{x}^{\prime} & =(C \sin \gamma x+D \cos \gamma x) e^{i k_{z} z}{ }_{e}^{i \omega t},
\end{aligned}
$$

Since no normal $\vec{B}$ components are allowed at $x=0, D=0$. Also, at $x=0$,

$$
B_{t}=\left(B_{z}^{\prime}+B_{z}^{0}\right) \hat{z} \text {, }
$$

so from

$$
\frac{\partial B_{t}}{\partial \hat{n}_{5}}=0
$$

you have $A=0$, sc

$$
\begin{aligned}
& B_{z}^{\prime}=B \cos \gamma \times e^{i k_{2} z} e^{i \omega t}: \\
& B_{x}^{\prime}=C \sin \gamma \times e^{i k_{z}{ }^{2}}{ }_{\mathrm{d}}^{i \omega t} .
\end{aligned}
$$

At the plasma boundary,

$$
\hat{n}_{\mathrm{s}}=-\left(\hat{\mathrm{x}}+\hat{z} \zeta_{0} k_{\mathrm{z}} \sin \mathrm{k}_{\mathrm{z}}{ }^{2} .\right.
$$

so for $\hat{n}_{\mathrm{s}} \cdot \overrightarrow{\mathrm{B}}=0$ you have

$$
\left.\left(\hat{x}+\hat{z} \zeta_{0} k_{z} \sin k_{z} z\right) \cdot\left\{\left(B_{z}{ }^{0}+B_{z}^{\prime}\right) \hat{z}+B_{x}^{\prime} \hat{x}\right]\right|_{x=q+5}=0
$$


Expanding the appropriate functions, you have

$$
\begin{aligned}
& \left.B_{x}^{\prime}\right|_{x=2+\xi}=\left.B_{x}^{\prime}\right|_{x=Q}+\left.\frac{\partial B}{\partial x}\right|_{x=e} \cdot \zeta . \\
& \left.B_{2}^{0}\right|_{x=\ell+\xi}=\left.B_{2}^{0}\right|_{x=\ell}+\left.\frac{\partial B_{2}^{0}}{\partial x}\right|_{x=\ell} \cdot \zeta . \\
& \left.R_{z}^{\prime}\right|_{x=2+j^{\prime}}=\left.B_{z}^{\prime}\right|_{x=Q}+\left.\frac{\partial B_{z}^{\prime}}{\partial x}\right|_{\{=R} \cdot 5 .
\end{aligned}
$$

Hence, to first order in $\zeta$ you have

$$
b_{x}^{\prime}=-r_{0} k_{z} \sin k_{z}(-1)^{n} B_{0}
$$

or

$$
C=-\frac{\zeta_{0} k_{2} \sin k_{2} z}{\sin \gamma^{R}} B_{0}(-1)^{n}
$$

Hence you have, from $\frac{\partial \mathbf{E}_{x}^{\prime}}{\partial x}=-\frac{\partial B_{2}^{\prime}}{\partial z}$. that the two fields are:

$$
\begin{aligned}
& B_{x}^{\prime}=-\xi_{0} k_{z} \sin k_{z} z B_{0}(-1)^{n} \frac{\sin \gamma x}{\sin \gamma l}, \\
& B_{z}^{\prime}=-\gamma \xi_{0} e^{i k_{2} z} B_{0}(-1)^{n} \frac{\cos \gamma x}{\sin \gamma l} .
\end{aligned}
$$

For physical reasons cited earlitr, we are considering

$$
\zeta_{0} k_{z}=2 \pi \frac{\zeta_{0}}{\lambda_{2}} \ll 1
$$

and the variation in field intensity is at $x=\ell$, to first order in $\zeta$.

$$
\frac{(B)^{2}}{8 \pi} \simeq \frac{\left(B_{z}{ }^{0}+B_{z}^{\prime}\right)^{2}}{8 \pi} \text {. }
$$

or

$$
\delta p_{i}=-\frac{B_{0}^{2}}{4 \pi} \gamma 5 \cot \gamma Q
$$

for

$$
\begin{aligned}
& E_{z}^{0}=0, \\
& B_{z}^{0}=\hat{z} B_{0} \cos \left(\frac{n \pi}{\ell}\right) x, \\
& E_{y^{0}}=\hat{y} B_{0} \sin \left(\frac{\pi \pi}{\ell}\right) x .
\end{aligned}
$$




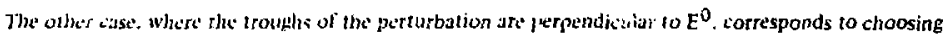

$$
\begin{aligned}
& B_{z}^{0}=0 \text { and } E_{x}^{0}=0 . \\
& E_{z}^{0}=\hat{B} E_{0} \sin \left(\frac{n \pi}{l}\right) x, \\
& B_{y}^{0}=\hat{y} B_{0} \cos \left(\frac{n \pi}{l}\right) x .
\end{aligned}
$$

Then followilg the sank̇ procedunt, but with

$$
\left(\hat{n}_{\mathrm{s}} \times\left.\vec{E}\right|_{\mathrm{s}=\imath+\xi}=0\right.
$$

and vanishing tangential field at $x=0$ and $\nabla \cdot E=0$ in interior, gives

$$
\begin{aligned}
& E_{z}^{\prime}=-E_{0}\left(\frac{1 n \pi}{l}\right)(-1)^{n} \zeta_{0} e^{i k_{z} z} \frac{\sin }{\sin \frac{\gamma x}{\gamma l} .} \\
& E_{x}^{\prime}=\frac{k_{z}}{\gamma^{\prime}}\left(\frac{n \pi}{Q}\right)(-1)^{n} \zeta_{0} \sin k_{z} z \frac{\cos \frac{\gamma x}{\sin } \frac{\gamma l}{\gamma l} .}{}
\end{aligned}
$$

So the B-field perturbation is along $y$ and given by

$$
\begin{aligned}
& \vec{B}^{t}=-\frac{c}{\omega} \nabla \times \vec{E}^{\prime} \\
& {\left[k_{0}=\frac{\omega}{c}=\frac{n \pi}{q}\right]} \\
& B^{\prime}=-\hat{y} B_{0}(-1)^{n} \zeta_{0} e^{i k_{z} z} \frac{\cos }{\sin \frac{\gamma x}{\gamma^{2}}} \frac{k_{0}^{2}}{\gamma}, k_{2}{ }^{2}<k_{0}
\end{aligned}
$$

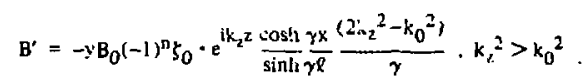

Again, $\delta p_{\mathrm{f}}=2 B^{0} B^{\prime}$ and the sign of the pressure change is the sane as in the previous case.

\section{PLASMA PARTICLE PRESSURE}

In the case of a skin density much less than core density, sound waves are restricted to small amplitudes because the large den'ity gradient to the core provides a large impedance mismarch. In the catse of the average confinement to core density, potentially large surface wayes can exist as modes of the plasma as a whole.

in cases where the time stale of the surface motion is fister thun the collision time for clic ians, the plasma belaves more like a cloud of noninteracting particles raining onto a moving boundary.

As will now be demonstrated, the stability eriteria are the sane for all the ahove cuses.

Case A: Sound-Wave Generation. In order that a plasma have a surface wave present at frejuency $v$.

$$
\zeta=\zeta_{0} e^{j k_{z} z} e^{10 \times r}
$$


of small amplitude $\zeta$, no larger than $\sim \delta$, at the aperage confinement density $N_{\mathrm{c}}$. tus pressure variations at the surface must be given as solutions of the linearized hydrodynamic equation, the velocity potantial ior which satisfies the wave equation, with solutions, for an infinite cylinder of plasma:

$$
\phi=-i \zeta_{0} e^{i k_{z}^{2}} e^{i v t} \frac{\nu}{\gamma_{s}} \frac{J_{0}\left(\gamma r_{0}\right)}{J_{0}\left(\gamma_{0}\right)}
$$

where $J_{0}=\frac{\partial}{\partial r} J_{0}$.

and hence pressure variations at the surface:

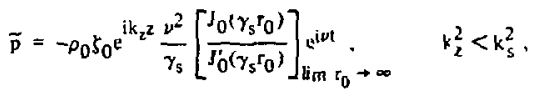

where, to recover the simpler geometry of the slab, we let $\gamma_{s} I_{0} \rightarrow \infty$. However, the $J_{0}$ are to be replaced by the $t_{0}$ if $k_{2}^{2}>k_{s}^{2}$.

$$
\begin{gathered}
\gamma_{s}=\sqrt{k_{z}^{2}-k_{s}^{2}}, \\
k_{s}^{2}=\left(\frac{\nu}{c_{s}}\right)^{2}, \\
c_{s}=\text { sound speed in the gos } . \\
\rho_{0}=\text { plosms density. }
\end{gathered}
$$

Case B: Noninteracting Particles. The pressuse variation at the density $\mathrm{N}_{i}^{\mathrm{c}}$ is

$$
P(t)-\bar{P}=4 N_{i}^{c} M_{i}\left\langle v_{2}^{j}\right\rangle \dot{\zeta}+N_{i}^{c} M_{i} \dot{\xi}^{2}
$$

We will ignore the square of the surface-wave velocity $\overline{\mathrm{P}}=$ average pressure $=2 \mathrm{~N}_{\mathrm{i}}^{\mathrm{c}} \mathrm{kt}$ and take the positive ditection of $\xi$ away from the wall into the plasma.

\section{DISPERSION RELATION FOR SURFACE WAVES}

For Case A, continujty of pressure across the plasma-radiation interface gives, for example,

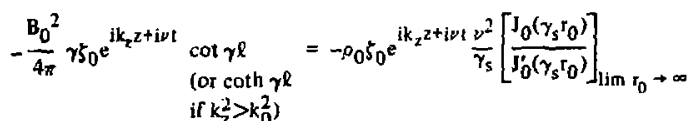

The oniy important point here is that stability requires $v^{2}$ to be real. The left side of the dispersion relation is all that affects this, with the requirement that

or

$$
\begin{gathered}
\cot \gamma \ell>0, k_{2}^{2}<k_{0}^{2} \\
\operatorname{coth} \gamma \ell>0, k_{2}^{2}>k_{0}^{2} .
\end{gathered}
$$


The coth function dows not change sign. so it is always true if $k_{\%}^{2}>k_{0}^{2}$. The other branch requires then that

$$
\gamma R<\pi / 2
$$

or

$$
N_{\pi} \sqrt{1-\frac{k_{2}^{2}}{k_{0}^{2}}}<\frac{\pi}{2}
$$

or

$$
\frac{k_{z}^{2}}{k_{0}^{2}}>1-\frac{1}{4 N^{2}} .
$$

To the extent thit a plasma can be represcnted as is collisional gas. characterized by a sound speed, perturbations with $\lambda_{2}=2 \pi / k_{2}<\lambda_{\text {radiation }}$ are stable. Longer wavelengths are unstable, $\lambda_{2}$ are modes of the butk plasma at irtipuency $\nu$.

For case B, the dispersion reiation is. ignoring the square of the surface velocity.

$$
\frac{-B_{0}^{2}}{4 \pi} \gamma \zeta\left(x_{t} t\right) \cot \gamma Q=4 N_{i}^{c} M_{i}\left\langle\gamma_{2}^{i}\right\rangle \frac{d \xi}{d t} .
$$

so

$$
\zeta=\zeta_{0}(0.2) \mathrm{e}^{-\alpha \mathrm{t}}
$$

with

$$
\alpha=\frac{B_{0}^{2}}{4 \pi} \frac{\gamma \cot \gamma R}{4 N_{i}^{c} M_{i}\left\langle r_{z}^{i}\right\rangle} .
$$

which tearly decilys for $\alpha>0$, which is the same criterion as in case $A$.

\section{ABLATIVE STABILITY}

Before it can be concluded that a plasma with linear dimensions $<\lambda_{\text {fgdiation }}$ is stable for all perturbations. it must be determined whether the rate of differential energy absorption due to the perturbation can exteed the diffusion of energy away from the heated retion. This requires a knowledge of the energy absorption of tihe plasma as a function or field intensity.

Stochastic heating is not a strong function of average piessure, but depends most jy upon the transit time of electrons through the skin. Skin-effect heating depends direstly upon the energy density at the corona. as Joes wave-particle heating.

One can make the following simple plausibility arguments for heating and diffusing.

First consider the skin effect: For a skin in which $v_{e}>\omega$. the energy flux is. per unit ared.

$$
\dot{S} ₹ P_{\mathrm{f}} \boldsymbol{\omega} \delta \quad P_{\mathrm{f}}=\frac{\mathrm{B}^{2}}{8 \pi} .
$$




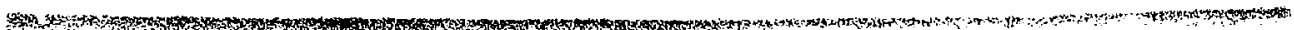

Hence energy is gdis. $d$ for the "stable" surface perturbation in repions protruding from the averuge surfise jwaty" from the plasma, corr.pared to those protruding inward, by the rate

$$
\Delta \dot{\mathrm{S}} \sim \Delta \mathrm{P}_{\mathrm{f}} \omega \mathbf{\delta} .
$$

but any perturbation satisfying the stability criterion $\left(\lambda_{2}<\lambda_{\text {Iadiation }}\right)$ has a time constant, ignoring local sinergy storage during the motion, of

$$
\tau_{\dot{s}}=\left(\frac{\Delta P_{i}}{54 N_{j}^{c} M_{j}\left\langle v_{z}^{i}\right\rangle}\right)^{-1}=\frac{4 N_{i}^{c} M_{i}\left\langle v_{2}^{j}\right\rangle}{B_{0}^{2} \gamma \cot \gamma l} 4 \pi,
$$

or, since $k_{2}>k_{0}, \gamma \simeq k_{2}$ so the time available for depositing heat is

$$
\tau_{\mathbf{s}^{\prime}}=\lambda_{\mathbf{z}} / \mathbf{v}_{2}^{\mathbf{j}}
$$

which is therefore always about the time to diffuse the heat across $\lambda z / 2$ even by ion conduction.

We conclude that the particle pressure does not rise faster than the field pressure in the perturbation. Since the wave-particle huatiug also only changes linearly witl intensity, the same conclusion holds. 


\section{Appendix E \\ I)ERIVATION OF THE SELF-CONSISTENT FIELD EQUATION FOR A MICROWAVE CORONA}

First divive the guiding-center constant of the motion $\mathrm{E}_{c}$.

$$
E_{\mathrm{c}}=\frac{1}{2} \mathrm{M}_{\mathrm{e}} \dot{\mathrm{R}}^{2}+\psi_{\mathrm{e}}-\mathrm{e} \phi=\text { colstant }
$$

where the actual coordinates are $(\vec{r}, \vec{v})$ :

$$
\begin{aligned}
& \vec{r}=\vec{R}+\vec{\rho}, \\
& \ddot{\vec{r}}=-t \overrightarrow{\mathrm{F}}(\overrightarrow{\mathrm{r}}, \mathrm{t})-\frac{\overrightarrow{\mathrm{v}}}{\mathrm{e}} \times \overrightarrow{\mathrm{B}}(\overrightarrow{\mathrm{r}}, \mathrm{t}), \\
& \psi_{\mathrm{e}}=\frac{\mathrm{e}^{2}|\mathrm{E}|^{2}}{4 \mathrm{H}_{\mathrm{e}} \omega^{2}}=\psi_{\mathrm{e}}(\overrightarrow{\mathrm{R}}) . \\
& |\mathrm{E}|=\text { magnitude of the peak } \overrightarrow{\mathrm{E}} \text { field. }
\end{aligned}
$$

$\vec{R}$ is a slowly varying past (guiding center) and $\vec{\rho}$ has time dependence $\omega^{-1}$.

letting the distribution function be, for electrons.

$$
f_{e, j}\left(\epsilon_{e, i}\right)=f_{e, j}\left(\frac{1}{2} M_{c, j} \dot{R}^{2}+\frac{e^{2} \mid E_{i}^{2}}{2 M_{e} \omega^{2}} \mp e \phi\right) \text {. }
$$

and rexixpossing this argument in terms of the orbit variables.

$$
f_{s}\left(\epsilon_{v}\right)=f_{e}\left\{\frac{l}{2} M_{e}\left(\vec{v}+\frac{e}{M_{e}} \int^{\prime} \vec{E} d t^{\prime}\right)^{2}+\psi_{e}\left[\vec{r}+\frac{c}{M_{e}} \int^{\prime} \int^{t^{\prime}}\left(E d t^{\prime \prime} d t^{\prime}\right)\right]-e \psi\right\} .
$$

where the approximate value for $\vec{\rho}$ of

$$
\vec{\rho}=\frac{\vec{t}}{M_{c}} \int^{t} \int^{t^{\prime}} E\left(\vec{R} \cdot t^{\prime \prime}\right) d t^{\prime \prime} d t^{\prime}
$$

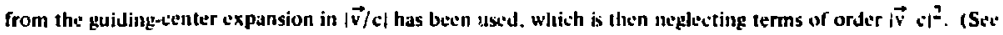
Motz and Watson. p. 164.1

Front

$$
\nabla \times \vec{E}=-\frac{1}{i} \frac{\partial \vec{B}}{\partial t} \operatorname{ind} \nabla \times \vec{B}=\frac{4 \pi}{t} \vec{J}+\frac{1}{i} \frac{\partial \vec{E}}{\partial t}
$$

ubtain for time harmonis $\overrightarrow{\mathbf{E}}$.

$$
\nabla \times \nabla \times \vec{E}=\left(\frac{\omega}{c}\right)^{2}\left(\vec{E}-\frac{d \vec{r}}{\omega^{2}} \frac{\partial}{\partial t} \vec{J}_{q}\right)
$$

whers:

$$
\vec{J}_{c}=\int_{-\infty}^{+\infty}\left(f_{j}-f_{c}\right) \vec{v} d \vec{v} .
$$


Now look at $f_{e}$ (or $f_{i}$ ) term in $\vec{j}$,

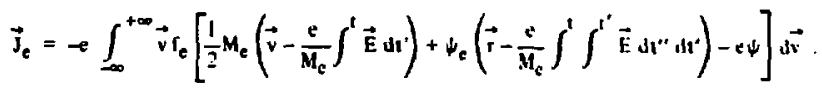

The essential step of the guidingerenter theory is to remove the assumed small. time-depundent part of the argument for $\psi_{\mathrm{e}}$ so the equation for $\overrightarrow{\mathrm{J}}_{\mathrm{e}}$ can be written, after making the variable transformation

$$
\begin{gathered}
\prime \vec{\eta}=\vec{v}+\frac{e}{M_{e}} \int^{\prime} \vec{E} d t^{\prime} . \\
\vec{\jmath}_{e}=-\int_{-\infty}^{+\infty}\left(\vec{\eta}-\frac{s}{M_{e}} \int^{\prime}\left(\vec{E} d t^{\prime}\right) r_{e}\left[\frac{1}{2} M_{e} \eta^{2}+\nu_{e}(\vec{r})-\infty\right] \vec{\eta} .\right.
\end{gathered}
$$

which, fro in obvious symmetry properties, yields

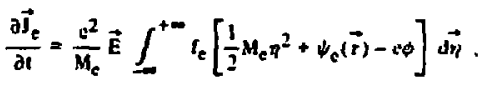

which, after normalizing $\mathrm{f}_{c}$ so $\mathrm{N}_{0}$ in the plasma interior. yields finally

$$
\frac{\partial \vec{J}_{v}}{\partial t}=\frac{t^{2}}{M_{c}} N_{0} \vec{E} \int_{-\infty}^{+\infty} r_{e} d \vec{v}
$$

After doing the strike for ions, this gives

$$
\nabla \times \nabla \times \vec{E}=\left(\frac{\omega}{c}\right)^{2}\left[1-\frac{\omega_{p e}^{2}}{\omega^{2}} \int_{-\infty}^{+\infty} c_{c} d \vec{\eta}-\frac{\omega_{p i}^{2}}{\omega^{2}} \int^{+\infty} r_{i} d \vec{\eta}\right] \vec{E} .
$$

Finally, if there is any reason to choose $f_{e, i}$ as Muxwellians, and after making the qujsi-neutral approximation, $N_{c}-N_{i}=N_{0}$, so that

$$
\otimes=\frac{e^{2}|E|^{2}}{8 M_{e} \omega^{2}}=\frac{\nu_{e}}{2}
$$

ignoring the $M_{e} / M_{i}$ em. Then, for instance

$$
\frac{i}{N_{0}} \int_{0}^{+\infty} r_{e} d \vec{\eta}=\operatorname{sxp}\left(-\frac{e^{2} E^{2}}{8 M_{\varepsilon} \omega^{2} K T}\right)=\frac{N_{c}}{N_{0}}
$$

and ignoring the ion current, you obtain quasi-neutral solution.

$$
\nabla \times \nabla \times \vec{E}=\left(\frac{\omega}{c}\right)^{2}\left[1-\frac{\omega_{p e}^{2}}{\omega^{2}} \exp \left(-\frac{c^{2} E^{2}}{8 M_{c} \omega^{2} K T}\right)\right] \vec{E} .
$$

We now briefly consider the solutions without the us: of guiding-eenler approximation or qunst-neutrality.

$-38-$ 
The ruasi-meutral tauation above is used as the beginning point, but more generally,

$$
\begin{gathered}
\nabla \times \nabla \times \vec{E}=\left(\frac{\omega}{c}\right)^{2}\left(\vec{E}-\frac{4 \pi}{\omega^{2}} \frac{\partial}{\partial t}\right), \\
\nabla^{2} \phi=4 \pi e\left(\left(f_{e}-f_{i}\right) d \vec{v}=4 \pi e\left(N_{e}-N_{j}\right) .\right. \\
H_{e}=-e \phi+\frac{(\vec{P}+e \vec{A})^{2}}{2 M_{e}} \text { (neglecting interparticle scattering in the skin) }, \\
\frac{\partial \vec{I}}{\partial t}=\vec{E}\left[\omega_{p e}^{2} \exp \left(\frac{-e^{2} E^{2}}{4 M_{e} \omega^{2} K T_{e}}+\frac{e \phi}{K T_{e}}\right)+\omega_{p i}^{2} \exp \left(\frac{-e^{2} E^{2}}{4 M_{i} \omega^{2} K T_{i}}-\frac{e \phi}{K T_{i}}\right)\right] . \\
\nabla^{2}=4_{0} N_{0} e\left[\exp \left(\frac{-e^{2} E^{2}}{4 M_{e} \omega^{2} K T_{e}}+\frac{e \phi}{K T_{e}}\right)-\exp \left(\frac{-e^{2} E^{2}}{4 M_{i} \omega^{2} K T_{i}}-\frac{e \phi}{K T_{i}}\right)\right] .
\end{gathered}
$$

Using for Cycle $1 N_{0}^{e, i}$ and $\frac{\partial}{\partial t} \vec{\jmath}$ from the Maxwellian quasi-neutral solution above, with corresponding $E_{0}, \phi_{0}$. solve for $N_{1}^{e, i}, \vec{j}_{1}^{e, i}, \vec{E}_{1}, \phi_{l}$, by computing from (C) the $N_{1}^{e, i}, j_{1}^{e, j}$ using $E_{0}, \phi_{0}$ in $H_{e}$ with a Fasor code. Then compute $\epsilon_{1}, \phi_{1}$ by solving $(A)$ and $(B)$ using $N_{1}^{e, i}, \vec{j}_{1}^{e, j}$. The values for $\epsilon_{1}$ and $\phi_{1}$ should now be used in $H_{e}$ for the second cysle, with $N_{2}^{e, i}, \vec{J}_{2}^{e}$ now coming from FASOR, and then used in ( $A$ ) and (B) to obtain $E_{2} \phi_{2}$. This procedure should converge to the selt-consistent fields, and has been observed to do so several times. (See

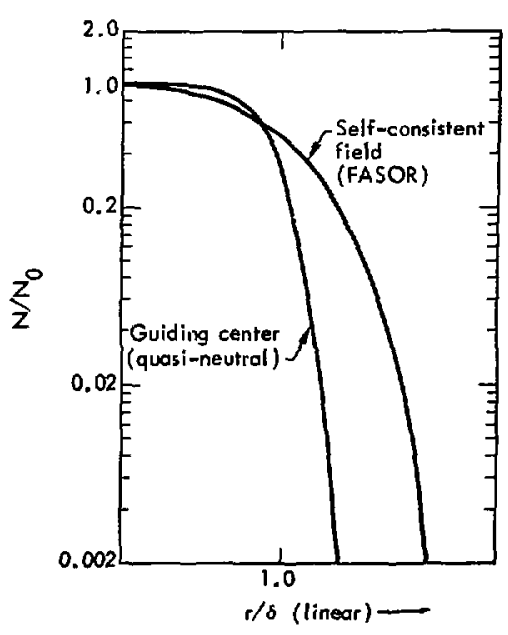

Fig. El.) FASOR is an acronym for Fast Orbit Integrator, which is a finite-difference code solving the electron motion in a given field and averaying the density according to transit time in a given zone.

Fig. E1. 


\section{Acknowledgements}

Much of this work was performed by the author in association with Controlled Fusion. Inc., San Rimon. California. The author would like to thank A. D. MacKay of CFI for carrying out detailed integrations in Appendix A, part 1: thanks also to L. D. Howard of CFI for machine calculations on particle oftiits.

\section{- Bibliography}

Boyd, T. J. M., and J. J. Sanderson, Plasma Dimamics (Barnes \& Noble, New York. 1969 ).

Ensley, D. L., Research and Detelopment Proposal for Mtcruwave Confinement of Plasma. Lawrence Liverinore Laboratory Rropt. UCIR-824 (July 12, 1974).

Jackson. J. D., Classical Electroḍnamlcs (John Wiley \& Sons, New York, 1962).

Marcuse. D., "Stimulated Emission of Bremsstrahlung," Bell System Tech. J. 4I. 1557 (1962).

. "A Further Discussion of Stimulated Emission of Bremsstrahtung." Bell System Tech. J. 42. 415 (1963).

Morse, P. M., and H. Feshbach. Methuds of Theoretical Physics (McGraw-Hill. New York, 1953). pp. $1052-1056$.

Motz, H., and C. J. H. Watson, “The Radio Frequency Confinement and Acceleratior. if Plasmas," in Advances in Electronics and Elcciran Plýsics, L. Marton, Ed., vol. 23 (Academic Press, New York, 1967).

Sagdeev. R. 2., "Containment of a Plasma by the Pressure of a Standing Electromagnetic Wave," in Plasma Physics and the Problem of Controlled Thermonuclear Reactions, vol. 3, M. A. Leontovich, Ed. (Pergamon Press, London, 1959), p. 406.

Shafranov, V. D., "The Stability of a Cylindrical Guseous Conductor in a Magereir Fiejd," Sou, J. AL. Entergy' l. 709 (1956).

Trubnikov, B. A., "Particle Interactions in a Fully Jonized Pasma," in Reviews of Plasma Physics. vol. 1,

M. A. Leontovich, Ed. (Consultants Bureau, New York, 1965), pp. 105-204.

Volkov, T. F., "Oscillations and Stability of the Surlace of a Plasma in the Fielut of a Travelling Elictromagnetic: Wave," in Plasma Phystis and the Problem of Controlled Thermonuclear Reactions, vol 4, M. A. Leontovich. Ed. (Pergamon Press, London. 1960), p. 127.

Weibel. E. S., "Dynamic Stabilization of a Plasma Colunn," Phys. Fluids 3, 946 (1960). 